\title{
Über Zugriffspfade in Printwörterbüchern. Ein Beitrag zur Schnittstelle von Benutzungshandlungen und Wörterbuchform
}

\author{
Herbert Ernst Wiegand, Germanistisches Seminar, Universität Heidelberg, \\ Heidelberg, Bundesrepublik Deutschland \\ (herbert.ernst.wiegand@gs.uni-heidelberg.de)
}

Zusammenfassung: Nach der Einführung der terminologischen Adjektive adkurrent, inkurrent, binnenkurrent und exkurrent sowie einer Reihe von lexikographietheoretischen Begrifflichkeiten werden Zugriffspfade z.T. anhand von Beispielen erklärt und zahlreiche Zugriffspfadtypen unterschieden. Eine grundlegende Unterscheidung ist die zwischen äußeren und inneren Zugriffspfaden; bei den äußeren Zugriffspfaden werden einfache und zusammengesetzte äußere Zugriffspfade, bei den einfachen Zugriffspfaden mediostrukturelle und nichtmediostrukturelle äußere Zugriffspfade differenziert; weiterhin werden bei den mediostrukturellen binnenkurrente, verzeichnisexkurrente und wörterbuchexkurrente äußere Zugriffspfade unterschieden, sowie bei den nichtmediostrukturellen Zugriffspfaden die adkurrenten, binnenkurrenten und wörterbuchexkurrenten äußeren Zugriffspfade. Der Beitrag stellt insgesamt begriffliche Differenzierungen und eine dazugehörige Terminologie zur Verfügung, mit deren Hilfe die Datenakzessivität von Printwörterbüchern systematisch evaluiert und geplant sowie ihr mediostrukturelles Profil systematisch dargestellt werden kann.

Stichwörter: ADKURRENTER ÄUßERER ZUGRIFFSPFAD, AKZESSIVE ANGABE, ÄUßERE ZUGRIFFSSTRUKTUR, ÄUßERES ZUGRIFFSTEXTELEMENT, BINNENKURRENTER ÄUßERER ZUGRIFFSPFAD, DATENAKZESSIVITÄT, EINTRAGSEINGANG, EXKURRENTER ÄUSSERER ZUGRIFFSPFAD, EXTERNE VERWEISBEFOLGUNGSHANDLUNG, EXTERNE ZUGRIFFSHANDLUNG, INKURRENTER ÄUSSERER ZUGRIFFSPFAD, INNERE ZUGRIFFSSTRUKTUR, MEDIOSTRUKTURELLE ORIENTIERUNG, ORDNUNGSSTRUKTUR, VERWEISVERMITTELNDES TEXTSEGMENT, ZUSAMMENGESETZTER ÄUSSERER ZUGRIFFSPFAD

\begin{abstract}
About Access Routes in Printed Dictionaries. A Contribution towards the Intersection of Use Procedures and Dictionary Form. Following the introduction of the terminological adjectives adcurrent, incurrent, inner-current and excurrent as well as a series of lexicographic-theoretical concepts, access routes are explained, partly by using examples, and a variety of types of access routes are distinguished. A basic distinction is that between outer and inner access routes. Outer access routes are divided into single and complex ones with single access routes being divided into mediostructural and non-mediostructural outer access routes. Mediostructural outer access routes are divided into inner-current, table of contents excur-
\end{abstract}


rent and dictionary excurrent outer access routes whilst non-mediostructural access routes are divided into adcurrent, inner-current and dictionary excurrent outer access routes. This article presents conceptual differentiations and a relevant terminology with which the data accessibility of printed dictionaries as well as their mediostructural profile can be evaluated and planned systematically.

Keywords: ACCESSIBLE ITEM, ADCURRENT EXTERNAL ACCESS ROUTE, COMBINED OUTER ACCESS ROUTE, CROSS-REFERENCE ENABLING TEXT SEGMENT, DATA ACCESSIBILITY, ENTRY ENTRANCE, EXCURRENT OUTER ACCESS ROUTE, EXTERNAL CROSSREFERENCE FOLLOW-UP PROCEDURE, INCURRENT OUTER ACCESS ROUTE, INNER ACCESS STRUCTURE, INNER-CURRENT OUTER ACCESS ROUTE, MEDIOSTRUCTURAL ORIENTATION, ORDERING STRUCTURE, OUTER ACCESS PROCEDURE, OUTER ACCESS STRUCTURE, OUTER ACCESS TEXT ELEMENT

\section{Zur Schnittstellen- und Pfadmetaphorik}

Unter einer Schnittstelle wird in unterschiedlichen Bereichen etwas Verschiedenes verstanden. Die Schnittstelle zwischen einem Benutzer-in-actu und dem Printwörterbuch, das er benutzt, ist eine Menge von lexikographischen Schnittstellenelementen, die bestimmte Benutzungshandlungen ermöglichen bzw. erforderlich machen. Bei einem Printwörterbuch als einem statischen Informationssystem sind alle Schnittstellenelemente, die hier interessieren, Komponenten der Wörterbuchform, und zwar besonders solche, die externe Zugriffshandlungen ermöglichen sowie externe Verweisbefolgungshandlungen erforderlich machen und damit die externe lexikographische Datenakzessivität garantieren (vgl. Wiegand 2005). Dies sind vor allem die äußeren Zugriffsstrukturen in ihren unterschiedlichen Ausprägungen sowie die verweisvermittelnden Textsegmente in ihren vielseitigen Formen (vgl. dazu Wiegand 2002). Beim Benutzer ist die Beherrschung der allgemeinen Wörterbuchbenutzungspraxis (sensu Wiegand 1998: 364ff) die wesentliche Voraussetzung dafür, dass die Schnittstelle funktioniert.

In einer bestimmten Perspektive können die Ausführungen von Benutzungshandlungen als Bewegungen eines Benutzers-in-actu zu einem Wörterbuch und innerhalb eines Wörterbuchs beschrieben werden, die jeweils bestimmte individuelle Pfade etablieren. Liest Werner beispielsweise einen Text und versteht ein Wort $W_{1}$ nicht, so dass er daraufhin $\mathrm{zu}$ einem Wörterbuch $\mathrm{Wb}_{1}$ greift und eine externe Zugriffshandlung erfolgreich ausführt, dann kann man z.B. sagen: Werner hat einen Zugriffspfad etabliert, auf dem er von $W_{1} \mathrm{zu}$ $\mathrm{Wb}_{1}$ gelangt ist. Erschließt Werner in einem Wörterbuchartikel anhand eines verweisvermittelnden Textsegmentes einen Verweis und folgt diesem dadurch, dass er eine Verweisbefolgungshandlung erfolgreich ausführt, so dass er zum Verweiszielbereich kommt, so kann man z.B. sagen: Werner hat einen wörterbuchinternen Zugriffspfad etabliert, auf dem er vom verweisvermittelnden Textsegment zum Verweiszielbereich gelangt ist. 
In diesem Beitrag wird es darum gehen, alle Typen von Zugriffspfaden unter einer einheitlichen Terminologie kennen zu lernen. Bei der Terminologiebildung greifen wir auf folgende lateinische Verben zurück: currere (,laufen'), incurrere (,hineinlaufen'), excurrere (,hinauslaufen') und adcurrere (,bis zu einem bestimmten Punkt laufen'). Nach diesen Verben bilden wir folgende fachsprachliche Adjektive: binnenkurrent, inkurrent, exkurrent und adkurrent. Die vier Adjektive sind nicht steigerungsfähig; sie sind attributiv, prädikativ und adverbiell verwendbar. Anhand der Veranschaulichung in Abb. 1-1 lässt sich die Bedeutung dieser vier Adjektive leicht erfassen.

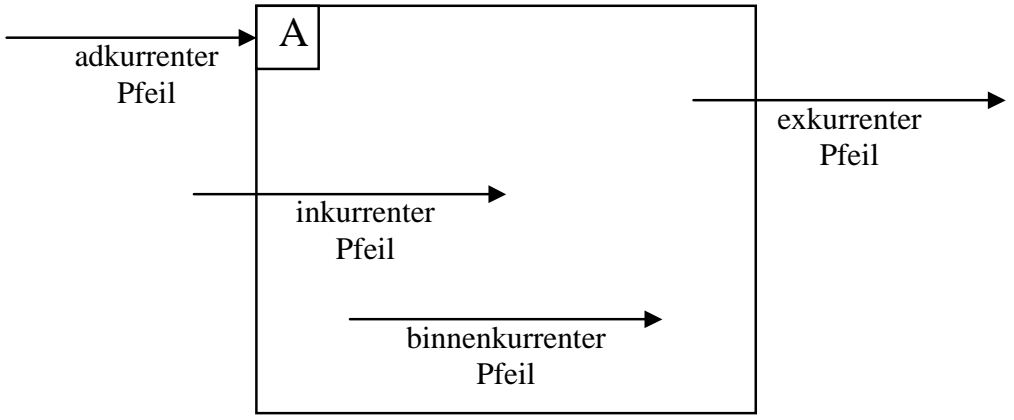

Abb. 1-1: Veranschaulichung zur Semantik der Terminologie

Ein adkurrenter Pfeil führt hin zu etwas. Ein inkurrenter Pfeil führt in etwas hinein. Ein binnenkurrenter Pfeil verläuft innerhalb von etwas, und ein exkurrenter Pfeil führt aus etwas hinaus. Es wird also jeweils nur eine Eigenschaft der Pfeile benannt, so dass offen bleibt, welche der anderen Eigenschaften ein Pfeil auch noch hat. Bevor wir die erläuterten Termini auf Zugriffspfade beziehen, werden zunächst im 2. Abschnitt einige lexikographietheoretische Begrifflichkeiten eingeführt, deren Kenntnis zum Verständnis der späteren Ausführungen im 3. Abschnitt erforderlich ist.

\section{Zu einigen Schnittstellenelementen und Benutzungshandlungen}

Jedes Printwörterbuch weist mindestens eine äußere Zugriffsstruktur auf. Eine äußere Zugriffsstruktur ist eine lineare Ordnungsstruktur (im mathematischen Sinn), deren Elemente äußere Zugriffstextelemente sind, auf die aufgrund der intuitiven Kenntnis der Ordnungsstrukturgesetzlichkeiten extern zugegriffen werden kann (vgl. Wiegand 2005). Jede äußere Zugriffsstruktur weist einen direkten Zugriffsbereich auf. In diesem liegen die direkt akzessiven lexikographischen Daten. Im Schriftkulturraum der Alphabetschriften sind die alphabetischen makrostrukturellen äußeren Zugriffsstrukturen die bekanntesten und die wichtigsten. Ihr direkter Zugriffsbereich sind Wörterverzeichnisse; ihre 
äußeren Zugriffstextelemente heißen unter makro- und zugriffsstrukturellem Aspekt Lemmata und unter dem Aspekt, dass mit ihnen etwas angegeben wird, lemmatische Angaben. Zu jedem Lemma gehört ein direkter Zugriffssektor, auf den es Zugriff eröffnet. Dieser Zugriffssektor heißt Wörterbuchartikel. Wörterbuchartikel sind die wichtigsten lexikographischen akzessiven Einträge. Andere akzessive Einträge sind die akzessiven Außentext-, Binnentext-, Umtext-, Einschub- und die akzessiven Registereinträge. Die äußeren Zugriffstextelemente dieser akzessiven Einträge (die den Lemmata entsprechen) sind Eintragseingänge und heißen z.B. Außentexteingang oder Umtexteingang. Ein Umtexteingang ist ein Element einer äußeren Umtextzugriffsstruktur; sein $\mathrm{Zu}$ griffssektor ist der zugehörige akzessive Umtexteintrag. Für die Eingänge, die $\mathrm{zu}$ anderen Typen gehören, gilt Entsprechendes. Äußere Zugriffsstrukturen stellen die externe Datenakzessivität für diejenigen potentiellen Benutzer sicher, die die allgemeine Wörterbuchbenutzungspraxis beherrschen und daher in der Lage sind, externe Zugriffshandlungen auszuführen.

Nach der in Wiegand (1998) entworfenen Theorie der Benutzungshandlungen wird eine Benutzungshandlung $h_{1}$, die zu Handlungstyp $T_{1}$ gehört, stets dadurch ausgeführt, dass mindestens eine andere Benutzungshandlung $\mathrm{h}_{2}$ ausgeführt wird, die zu einem bestimmten anderen Handlungstyp $T_{2}$ gehört; man sagt dann, dass $h_{2}$ im Ausführungsbereich von $h_{1}$ liegt. Beispielsweise wird eine Nachschlagehandlung dadurch ausgeführt, dass zuerst eine externe Zugriffshandlung und dann z.B. eine interne Zugriffshandlung ausgeführt wird. Eine externe Zugriffshandlung wird dadurch ausgeführt, dass eine externe Suchhandlung ausgeführt wird; und letztere wird dadurch ausgeführt, dass der Benutzer-in-actu als Zugriffshilfe die gleichen Ordnungsgesetzlichkeiten bemüht wie der Lexikograph, der die zugriffsstrukturelle Ordnung etabliert hat. Externe Zugriffshandlungen liegen nicht nur im Ausführungsbereich von Nachschlagehandlungen, sondern auch in dem von externen Verweisbefolgungshandlungen.

Der externen Datenakzessivität steht die interne gegenüber. Erstere ist obligatorisch. Letztere ist fakultativ. Die interne Datenakzessivität wird über innere Zugriffsstrukturen hergestellt. Nur die alphabetischen inneren Zugriffsstrukturen, die sich z.B. bei den alphabetisch geordneten Literaturangaben am Ende von Fachwörterbuchartikeln finden, funktionieren auf die gleiche Weise wie externe Zugriffsstrukturen. Denn die nichtalphabetischen inneren Zugriffsstrukturen basieren nicht auf allgemeinen Ordnungsstrukturen und deren intuitiver Kenntnis, sondern auf dem Wissen, das ein kundiger Benutzer über die textuellen Strukturen der Wörterbuchartikel eines bestimmten Wörterbuchs und gegebenenfalls über die der anderen akzessiven Einträge hat.

Die wichtigsten lexikographischen Daten sind in Wörterbüchern mit kondensierten Wörterbuchartikeln (sensu Wiegand 2003) die artikelinternen Angaben; wir berücksichtigen im Folgenden jedoch auch Angaben in den anderen akzessiven Einträgen, wie z.B. Umtextangaben und Registerangaben (vgl. Wiegand 2005). Hinsichtlich der Angabenakzessivität lässt sich eine ganze Reihe 
von Angabetypen unterscheiden. Die allermeisten Angaben gehören zum Typ der akzessiven Angabe. Eine Angabe ist dann akzessiv, wenn sie in einem der lexikographischen akzessiven Einträge steht, also z.B. in einem Wörterbuchartikel oder in einem akzessiven Umtexteintrag. Eine Angabe gehört zum Typ der nichtakzessiven Angabe, wenn sie z.B. in den Benutzungshinweisen zu Erklärungszwecken erwähnt wird. Eine Angabe gehört zum Typ der extern akzessiven Angabe, wenn sie von einem Benutzer, der von außerhalb des Wörterbuchs kommt, durch die Ausführung von externen Zugriffshandlungen erreichbar ist, die entweder im Ausführungsbereich von Nachschlagehandlungen oder in dem von Verweisbefolgungshandlungen liegen. Alle Angaben in allen Wörterbuchartikeln eines alphabetischen Wörterbuchs gehören zum Typ der extern direkt akzessiven Angabe: Auf sie kann ein Benutzer, der von außerhalb des Wörterbuchs kommt, über eine äußere Zugriffsstruktur, nämlich die alphabetische makrostrukturelle äußere Zugriffsstruktur, direkt zugreifen. Alle Lemmata als die äußeren Zugriffstextelemente und damit alle lemmatischen Angaben gehören zum Typ der primär akzessiven Angabe: Sie werden vom Benutzer-in-actu zuerst erreicht. Alle nichtlemmatischen Angaben gehören zum Typ der sekundär akzessiven Angabe: Sie sind nur über das Lemma und im kondensierten Wörterbuchartikel nach ihm erreichbar. Entsprechendes gilt für die anderen akzessiven Einträge, die keine Wörterbuchartikel sind: Die Eintragseingänge sind primär akzessiv; die eingangsexternen Angaben sind sekundär akzessiv. Zum Typ der extern indirekt akzessiven Angabe gehören Angaben, die für einen Benutzer, der von außerhalb des Wörterbuchs kommt, nur registervermittelt erreichbar sind, weil im Register zunächst auf ihre Fundortdaten zugegriffen werden muss. Angaben, die sowohl direkt über eine äußere Zugriffsstruktur als auch registervermittelt erreichbar sind, gehören zum Typ der extern direkt und indirekt akzessiven Angabe. Den extern akzessiven stehen die extern nichtakzessiven Angaben gegenüber. Letztere finden sich in Wörterbüchern, die hinsichtlich der Angabenakzessivität Defekte aufweisen. Dies ist z.B. im Dornseiff (1934) der Fall. Dieser weist, im Unterschied zum Dornseiff (2004), kein vollständiges alphabetisches Zugriffsregister auf. Daher sind alle Angaben in den sachgruppenbezogenen Wörterbuchartikeln, die nicht als Registereingänge genannt werden, extern nichtakzessive Angaben. Extern nichtakzessive Angaben können intern akzessiv sein. - Zum Typ der intern akzessiven Angabe gehören alle Angaben, die für einen Benutzer-in-actu nur dann über eine äußere Zugriffsstruktur erreichbar sind, wenn er von einem zugriffsverschiedenen Ort innerhalb eines Wörterbuchs kommt; dies ist dann der Fall, wenn er einem Verweis folgt, oder wenn ein wörterbuchinternes konfliktives Textsegment der Grund für die Benutzungshandlung ist. Ein zugriffsverschiedener Ort, an dem ein verweisvermittelndes oder ein konfliktives Textsegment begegnet, ist stets in einem anderen akzessiven Eintrag situiert. Im Terminus intern akzessive Angabe ist intern daher wie wörterbuchintern zu lesen. Von den wörterbuchintern akzessiven Angaben müssen die eintragsintern akzessiven Angaben, wie z.B. die artikelintern oder 
umtextintern akzessiven Angaben, unbedingt unterschieden werden; denn die Letztgenannten heißen so, weil sie nicht über äußere, sondern über innere Zugriffsstrukturen erreichbar sind. Zum Typ der extern und intern akzessiven Angaben gehören alle Lemmazeichengestaltangaben, die Verweisadressenträger sind. Auf sie kann sowohl durch die Ausführung externer Zugriffshandlungen, die im Ausführungsbereich von Nachschlagehandlungen liegen, extern zugegriffen werden, als auch durch die Ausführung externer Zugriffshandlungen, die im Ausführungsbereich von Verweisbefolgungshandlungen liegen.

Angaben, die nur über eine äußere Zugriffsstruktur erreichbar sind, heißen monoakzessive Angaben. Sind sie von einem Benutzer-in-actu, der von außerhalb des Wörterbuchs kommt, über nur eine äußere Zugriffsstruktur erreichbar, dann liegen extern monoakzessive Angaben vor. Sind sie dagegen nur von einem Benutzer-in-actu, der von einem zugriffsverschiedenen Ort innerhalb eines Wörterbuchs kommt, über eine äußere Zugriffsstruktur erreichbar, liegen intern monoakzessive Angaben vor. Angaben, die über mehr als eine äußere Zugriffsstruktur erreichbar sind, heißen polyakzessive Angaben. Sind sie von einem Benutzer-in-actu, der von außerhalb des Wörterbuchs kommt, über mehrere äußere Zugriffsstrukturen erreichbar, dann liegen extern polyakzessive Angaben vor. Sind sie dagegen nur von einem Benutzer-in-actu, der von einem zugriffsverschiedenen Ort innerhalb eines Wörterbuchs kommt, über mehrere äußere Zugriffsstrukturen erreichbar, liegen intern polyakzessive Angaben vor.

Nach diesen Überlegungen kann der Typologiegraph in Abb. 2-1 präsentiert werden; da der Typologiegraph nicht kommentiert ist, sei ausdrücklich darauf hingewiesen, dass die unmittelbaren Untertypen des Typs der akzessiven Angabe nur stufen- aber nicht kriteriengleich sind.

Wir hatten bereits gesehen, dass die jeweilige Ausführung von Verweisbefolgungshandlungen in metaphorischer Sicht- und Redeweise als Bewegungen des Benutzers-in-actu innerhalb eines Wörterbuchs verstanden werden können. Damit solche Bewegungen zielgerichtet erfolgen können, weisen alle verweisvermittelnden Textsegmente, wie z.B. Verweisangaben oder Angaben mit Verweiskennzeichnung, eine mediostrukturelle Orientierung auf (vgl. Wiegand 2002); diese ist eine Eigenschaft anhand derer dem Benutzer ersichtlich ist, wo er den Verweiszielbereich findet. Sie ist dadurch gegeben, dass mit dem adressenvermittelnden Textsegment, das ein Teil des verweisvermittelnden Textsegmentes ist, die Verweisadresse genannt wird, die als mediostrukturelles Leitelement für die Ausführung einer Verweisbefolgungshandlung benötigt wird. In diesem Beitrag spielt besonders die mediostrukturelle Außenorientierung eine Rolle; diese liegt vor, wenn mit dem adressenvermittelnden Textsegment eine Verweisaußenadresse genannt wird. Verweisaußenadressen sind solche Verweisadressen, die nicht im gleichen akzessiven Eintrag zu finden sind, in dem die verweisvermittelnden Textsegmente stehen, mit dem sie genannt werden. Wird beispielsweise mit einer Verweisadressenangabe einer artikelinternen Verweisangabe eine Umtextaußenadresse genannt, die in einer 
äußeren Umtextzugriffsstruktur liegt, dann ist die gegebene mediostrukturelle Außenorientierung eine Umtextorientierung, und es liegt eine umtextorientierte Verweisangabe vor. Entsprechend gibt es u.a. artikelinterne binnentextorientierte, artikelorientierte und einschuborientierte Verweisangaben. Erschließt ein Benutzer-in-actu beispielsweise anhand einer umtextorientierten Verweisangabe einen Verweis, dann ist dieser ebenfalls umtextorientiert. Folgt er dem umtextorientierten Verweis dadurch, dass er eine externe Verweisbefolgungshandlung ausführt und ist diese erfolgreich, so dass er die Verweisadresse gefunden hat, dann hat der erfolgreiche Benutzer einen Zugriffspfad etabliert, der ebenfalls umtextorientiert ist. - Damit sind wir bei den Zugriffspfaden, die im nächsten Abschnitt z.T. anhand von Beispielen systematisch betrachtet werden.

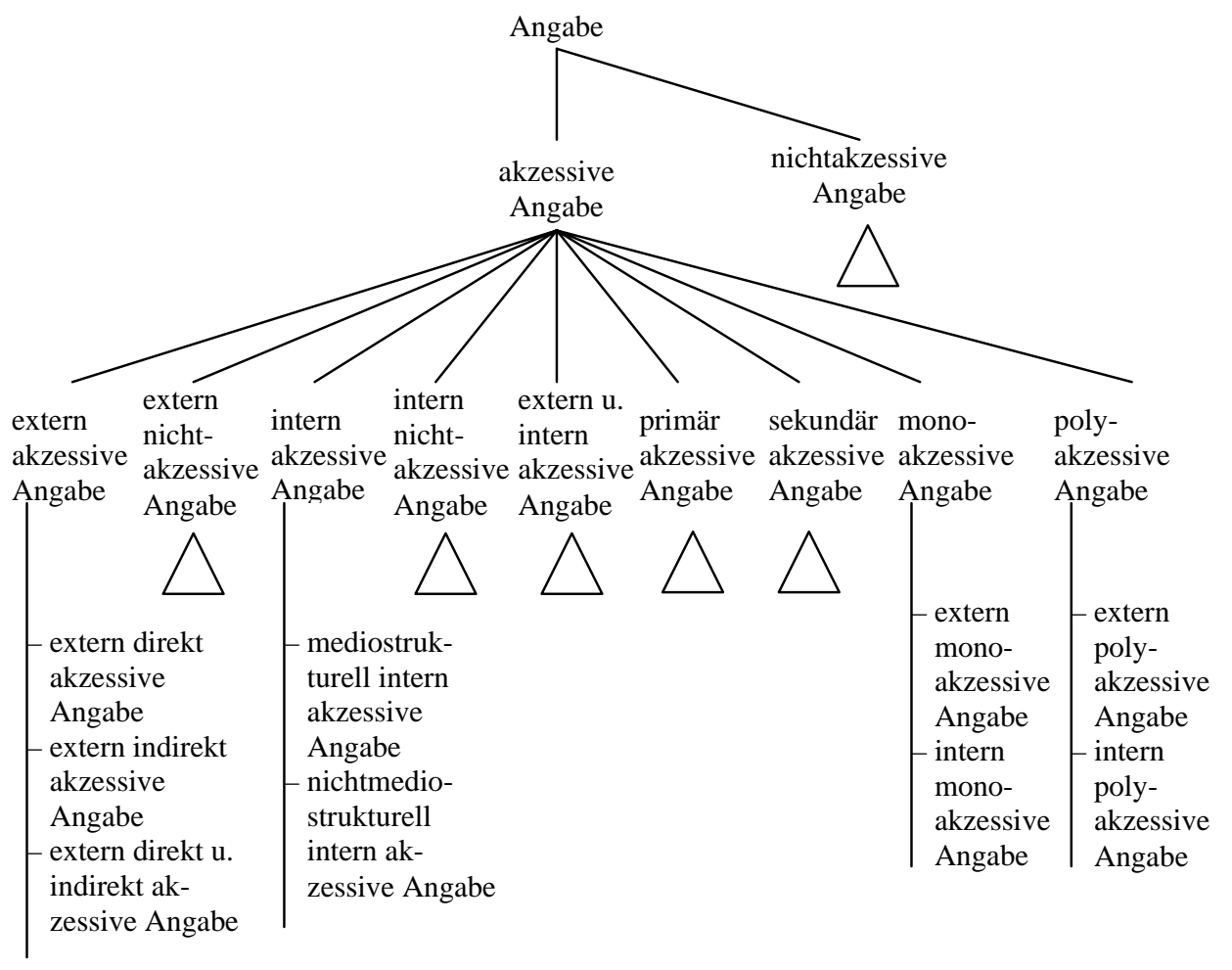

Abb. 2-1: Nichtkommentierter Typologiegraph zu einem Ausschnitt aus einer Typologie von Angaben nach der externen Angabenakzessivität

\section{Zugriffspfade in Printwörterbüchern}

Die wichtigste Voraussetzung dafür, dass Zugriffspfade von Benutzern-in-actu im Zuge der Ausführung von Zugriffshandlungen etabliert werden können, ist 
die Existenz von Zugriffsstrukturen; denn nur sie erlauben einen Zugriff auf lexikographische Daten, mit dem eine erfolgreich ausgeführte Zugriffshandlung abgeschlossen wird. Ein Zugriff ist das Ergebnis einer systematischen Suche bei der Ausführung von Zugriffshandlungen unter Zuhilfenahme von Ordnungsgesetzlichkeiten und von Wissen über die textuellen Strukturen in akzessiven Einträgen. Bei den Zugriffspfaden werden die einfachen Zugriffspfade von den zusammengesetzten unterschieden. Für Erstere kann folgende Definition (= D) gegeben werden:

(D 3-1: einfacher Zugriffspfad)

Ein einfacher Zugriffspfad ist ein von einem Benutzer-in-actu im Zuge der Ausführung einer erfolgreichen Zugriffshandlung etablierter Pfad, für dessen Pfadanfang und Pfadende folgende Bedingungen gelten:

(a) Ist der Pfadanfang wörterbuchextern, dann ist das Pfadende wörterbuchintern;

(b) Ist der Pfadanfang wörterbuchintern, dann ist das Pfadende entweder wörterbuchintern oder -extern.

Der Terminus einfacher Zugriffspfad wird oberbegrifflich verwendet, und zwar für einfache äußere und einfache innerer Zugriffspfade. Zusammengesetzte Zugriffspfade gibt es nur bei den äußeren Zugriffspfaden.

\section{1 Äußere Zugriffspfade}

Äußere Zugriffspfade haben ihren Namen daher, dass sie zu und durch Abschnitte von äußeren Zugriffstrukturen führen und nicht etwa daher, dass ein Benutzer von außerhalb des Wörterbuchs kommt. Es können einfache äußere von zusammengesetzten äußeren Zugriffspfaden unterschieden werden; wir betrachten zunächst die Ersteren. Auf die Letzteren wird in 3.1.2 eingegangen.

\subsubsection{Einfache äußere Zugriffspfade}

Einfache äußere Zugriffspfade sind diejenigen Zugriffspfade, die bei der Wörterbuchbenutzung am häufigsten etabliert werden. Ein einfacher äußerer Zugriffspfad kann wie folgt definiert werden:

(D 3-2: einfacher äußerer Zugriffspfad)

Ein einfacher äußerer Zugriffspfad ist ein benutzerspezifischer Weg, der durch eine Reihe von äußeren Zugriffstextelementen einer äußeren $\mathrm{Zu}$ griffsstruktur zu einem gesuchten äußeren Zugriffstextelement führt und der durch die erfolgreiche Ausführung einer externen Zugriffshandlung etabliert wird.

Ein einfacher äußerer Zugriffspfad hat stets zwei Pfadstationen: einen Pfadanfang und ein Pfadende. Ein Pfadanfang ist entweder der wörterbuchexterne 
textuelle Ort - wie im Beispiel B 3-1 - oder derjenige kognitive Zustand, der als Basis für die Erfassung des Leitelements dient, das ein Benutzer für die Ausführung einer externen Zugriffshandlung benötigt, die im Ausführungsbereich einer Nachschlagehandlung liegt; oder der Pfadanfang eines einfachen äußeren Zugriffspfades ist wörterbuchintern situiert. Dabei muss zwischen zwei Fällen unterschieden werden; der erste ist gegeben, wenn - wie in den Beispielen B 3-2 bis B 3-4 - der Pfadanfang ein verweisvermittelndes Textsegment ist, dessen adressenvermittelndes textuelles Teilsegment die Verweisadresse liefert, die als mediostrukturelles Leitelement dient. Der zweite Fall liegt vor, wenn - wie im Beispiel B 3-5 - der Pfadanfang ein konfliktives Textsegment ist, welches das Leitelement für die Ausführung einer Konsultationshandlung liefert, in deren Ausführungsbereich eine externe Zugriffshandlung liegt.

Nach diesen Erörterungen lassen sich zunächst die nachfolgend genannten Typen von einfachen äußeren Zugriffspfaden unterscheiden, wobei bei der Bildung der Termini für die Zugriffspfadtypen die in Abb. 1-1 erläuterten Adjektive verwendet werden.

(1) Der Typ des adkurrenten äußeren Zugriffspfades: Zu diesem Zugriffspfadtyp gehören alle einfachen Zugriffspfade, deren Pfadanfang wörterbuchextern und deren Pfadende ein äußeres Zugriffstextelement ist, so dass es wörterbuchintern situiert ist. Dieser Zugriffspfadtyp gehört zu den nichtmediostrukturellen Zugriffspfadtypen (vgl. 3.1.1.3).

(2) Der Typ des binnenkurrenten äußeren Zugriffspfades: Zu diesem Zugriffspfadtyp gehören alle einfachen Zugriffspfade, deren Pfadanfang und Pfadende wörterbuchintern situiert ist. Zum zweiten Typ gehören zwei Untertypen:

(2.1) Der Typ des mediostrukturellen binnenkurrenten äußeren $\mathrm{Zu}$ griffspfades: $\mathrm{Zu}$ diesem Zugriffspfadtyp gehören alle einfachen Zugriffspfade, deren Pfadanfang ein verweisvermittelndes Textsegment und deren Pfadende wörterbuchintern situiert ist.

(2.2) Der Typ des nichtmediostrukturellen binnenkurrenten äußeren Zugriffspfades: Zu diesem Zugriffspfadtyp gehören alle einfachen Zugriffspfade, deren Pfadanfang ein wörterbuchinternes konfliktives Textsegment und dessen Pfadende wörterbuchintern situiert ist.

In den folgenden Abschnitten werden zu den Typen (1) bis (2.2) Beispiele betrachtet. Dabei ist zu beachten, dass die vier bisher eingeführten Typen in einer Typologie der Zugriffspfade auf den obersten Typologiestufen stehen, so dass die Beispiele zugleich Beispiele für einen von deren Untertypen sind. In den Beispielüberschriften stehen die Termini für diese Untertypen hinter dem Schrägstrich. 


\subsubsection{Adkurrente äußere Zugriffspfade}

Systematisch betrachtet, gehören alle adkurrenten äußeren Zugriffspfade zu den nichtmediostrukturellen Zugriffspfaden, die später in Abschnitt 3.1.1.3 behandelt werden. Thre Betrachtung wurde jedoch vorgezogen: Nach meiner Einschätzung erleichtert ihre frühzeitige Erklärung das Verständnis der späteren Ausführungen.

Gegeben sei folgendes Beispiel:

(B 3-1: Adkurrenter äußerer Zugriffspfad/artikeladkurrenter äußerer Zugriffspfad)

Werner liest einen Text und stößt dort auf das Wort karamell. Mit der Suchfrage "Schreibt man das Adjektiv tatsächlich mit zwei l?" greift Werner zum Duden-2 ${ }^{2} \mathrm{GW}$ und findet dort den Artikel:

$$
\text { wa }{ }_{1} \text { : Ka|ra|mẹll, <indekl. Adj.>: bräunlich gelb. }
$$

Um das Lemma zu finden, hat Werner eine externe Zugriffshandlung mit dem Leitelement |karamell| ausgeführt, die erfolgreich war. Im Zuge der Ausführung dieser Benutzungshandlung hat er einen Zugriffspfad etabliert, der zu einem Untertyp des Typs des adkurrenten äußeren Zugriffspfades gehört, nämlich zum Typ des artikeladkurrenten äußeren Zugriffspfades. Zu diesem Untertyp gibt es auf der gleichen Typologiestufe mehrere kriteriengleiche Untertypen. Der entsprechende Ausschnitt aus einer Typologie der Zugriffspfade findet sich in Abb. 3-1.

Von den Definitionen, die zu dem Typologieausschnitt in Abb. 3-1 gehören, seien die beiden Folgenden angegeben:

(D 3-3: adkurrenter äußerer Zugriffspfad)

Ein adkurrenter äußerer Zugriffspfad ist ein nichtmediostruktureller einfacher äußerer Zugriffspfad, dessen Pfadanfang der jeweilige wörterbuchexterne textuelle Ort oder der jeweilige kognitive Zustand ist, der die Basis für die Erfassung des Leitelementes zur Ausführung einer externen Zugriffshandlung bildet und dessen Pfadende ein äußeres Zugriffstextelement ist.

(D 3-4: umtextadkurrenter äußerer Zugriffspfad)

Ein umtextadkurrenter äußerer Zugriffspfad ist ein nichtmediostruktureller einfacher äußerer Zugriffspfad, dessen Pfadanfang der jeweilige wörterbuchexterne textuelle Ort oder der jeweilige kognitive Zustand ist, der die Basis für die Erfassung des Leitelementes zur Ausführung einer externen Zugriffshandlung bildet, und dessen Pfadende ein Umtexteingang ist.

Der genaue Verlauf eines bestimmten konkreten Zugriffspfades, z.B. der des artikeladkurrenten äußeren Zugriffspfades, den Werner in B 3-1 etabliert hat, 
ist empirisch schwer festzustellen. Die beste Methode hierzu ist die der periaktionalen Benutzungskommentierung (sensu Wiegand 1998: 1015ff). Wendet man diese so an, dass man Testpersonen gleiche Zugriffsaufgaben stellt, dann zeigt sich, dass die konkreten Zugriffspfade im Detail sehr verschieden sind und nur darin übereinstimmen, dass der Pfadanfang und das Pfadende gleich sind. Es hat sich auch gezeigt, dass nur erfahrene Benutzer die kürzesten Zugriffspfade wählen, so dass sich möglichst kurze äußere Zugriffszeiten ergeben.

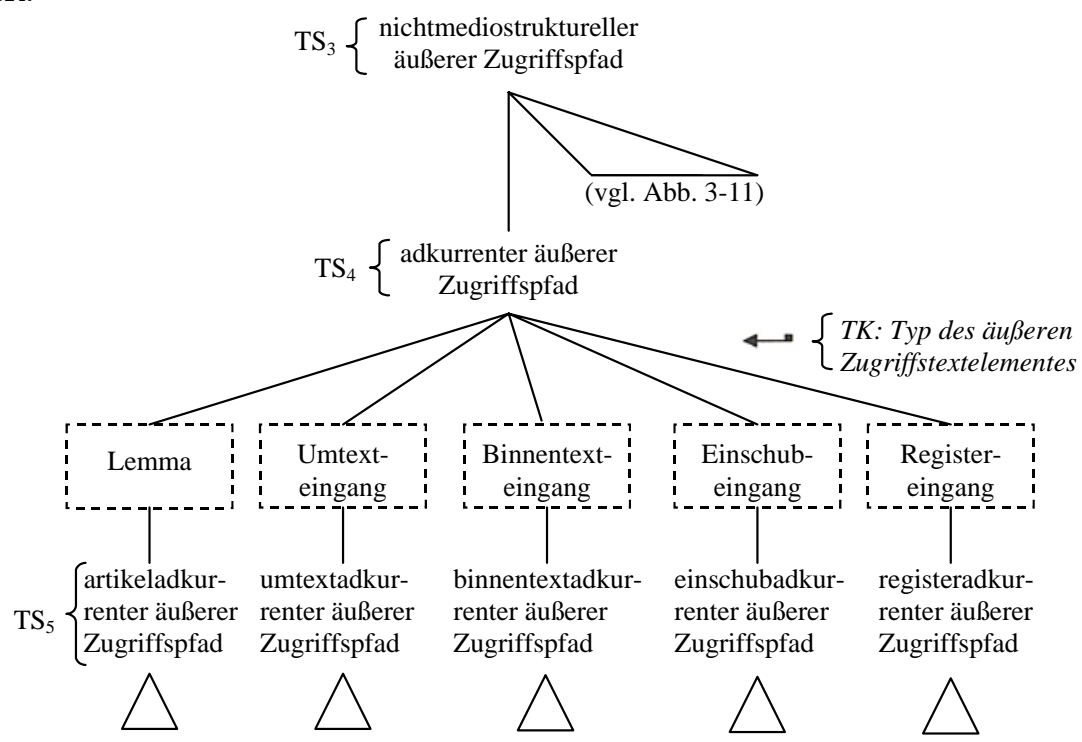

Abb. 3-1: Erster partiell erweitert kommentierter Typologiegraph zu einem Ausschnitt aus einer Typologie von Zugriffspfaden; Abkürzungen: TS = Typologiestufe; TK = Typologiekriterium; „« $\longleftarrow$ TK " bedeutet soviel wie die Anwendung des TK führt zu der Unterteilung

$\mathrm{Zu}$ jedem Zugriffspfadtyp lässt sich ein allgemeines Zugriffspfadbild angeben. Während alle allgemeinen Artikeltextstrukturbilder, Mikrostrukturbilder und Suchbereichsstrukturbilder formale Darstellungsmittel sind, die nach festen Konventionen gebildete Ausschnitte aus geordneten und kommentierten Baumgraphen darstellen, sind allgemeine Zugriffspfadbilder Veranschaulichungen, die allerdings ebenfalls nach festgelegten Darstellungskonventionen hergestellt sind (vgl. Wiegand 2007). Das allgemeine Strukturbild für den Typ des artikeladkurrenten äußeren Zugriffspfades findet sich in Abb. 3-2.

Wie die Abb. 3-2 zeigt, werden in einem allgemeinen Zugriffspfadbild der Pfadanfang, der Pfadverlauf durch einen Pfeil, das Pfadende sowie der durchlaufene Ausschnitt der zugehörigen äußeren Zugriffsstruktur repräsentiert, und die Bildelemente werden mit ihren Termini versehen. 


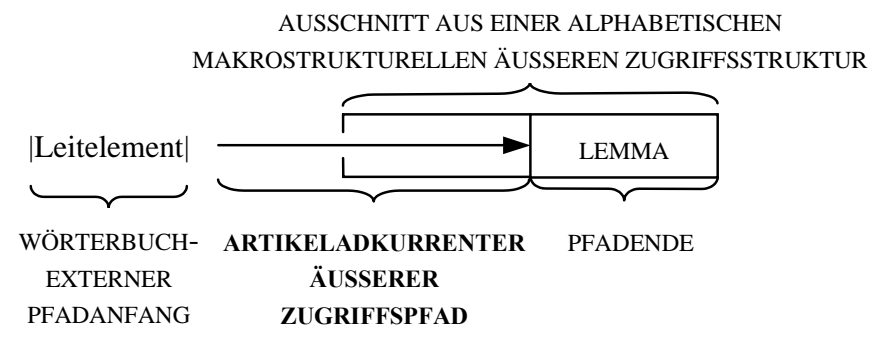

Abb. 3-2: Allgemeines Zugriffspfadbild für den Typ des artikeladkurrenten äußeren Zugriffspfades

\subsubsection{Mediostrukturelle äußere Zugriffspfade}

Bei den mediostrukturellen äußeren Zugriffspfaden werden hier nur die binnenkurrenten von den wörterbuchexkurrenten unterschieden. Weitere Unterscheidungen sind möglich, wenn man mehr als ein Wörterbuch zusammen betrachtet.

\subsection{Mediostrukturelle binnenkurrente äußere Zugriffspfade}

Bei den mediostrukturellen binnenkurrenten äußeren Zugriffspfaden ist die typologische Vielfalt am größten. Dies hängt damit zusammen, dass im Unterschied z.B. zu den adkurrenten äußeren Zugriffspfaden die wörterbuchinterne textuelle Lage des Pfadanfangs (woher der Pfad kommt), die mediostrukturelle Orientierung des Pfades und die textuelle Lage des Pfadendes (wohin der Pfad führt) bei der Typologiebildung Berücksichtigung finden.

Gegeben sei das folgende Beispiel:

(B 3-2: Binnentextexkurrenter Zugriffspfad/binnentextexkurrenter artikeladkurrent orientierter Zugriffspfad)

Werner studiert den eingelagerten Binnentext mit dem Binnentexttitel "Fach- und Sondersprachen" in ${ }^{2}$ Herders Sprachbuch. Ein Abschnitt des Binnentextes endet wie folgt:

„Andererseits sind Wörter aus dem technischen Bereich, die ursprünglich berufssprachliche Prägungen waren, in den allgemeinen Wortschatz eingegangen, weil die Dinge und Verfahren, die sie bezeichnen, den meisten Zeitgenossen bekannt geworden sind ( $>$ löten, $>$ schweißen, $>$ fräsen, $>$ Zarge, > Kardanwelle usw. $\square$ Wortschatz, $\square$ Wortbildung)."

Werner weiß nicht, was eine Zarge ist. Anhand der artikelorientierten Verweisangabe "\ Zarge" erschließt er einen artikelorientierten Verweis. Diesem folgt er dadurch, dass er unter Zuhilfenahme der Verweisadresse |Zarge| als mediostrukturellem Leitelement eine externe Verweisbefolgungshandlung dadurch 
ausführt, dass er eine externe Zugriffshandlung ausführt. Diese beendet er erfolgreich mit dem Zugriff auf das Lemma „Zar|ge“, das als Verweisadressenträger fungiert. Damit hat Werner einen Zugriffspfad etabliert, der zum Typ des binnentextexkurrenten artikeladkurrent orientierten Zugriffspfades gehört: Dieser ist einer der Untertypen (2. Stufe) des Typs des binnentextexkurrenten Zugriffspfades. - Im Folgenden betrachten wir ein weiteres Beispiel für einen mediostrukturellen binnenkurrenten äußeren Zugriffspfad.

(B 3-3: Artikelexkurrenter Zugriffspfad/artikelexkurrenter umtextadkurrent orientierter Zugriffspfad)

Werner konsultiert folgenden Artikel aus dem DCE:

wa 2 : darts /da:ts $\|$ darts / $\mathrm{n}[\mathrm{U}]$ any of several games in which DARTs ${ }^{1}$ (1) are thrown at a DARTBOARD

Anhand der umtextorientierten reduzierten Verweisangabe " $\mathrm{U}^{\prime \prime}$ erschließt er einen umtextorientierten Verweis; unter Zuhilfenahme der Umtextaußenadresse $|\mathrm{U}|$ als mediostrukturellem Leitelement führt er eine externe Verweisbefolgungshandlung dadurch aus, dass er eine externe Zugriffshandlung ausführt, die mit dem Zugriff auf den Umtexteingang " $\mathrm{U}^{\prime \prime}$ in der äußeren Umtextzugriffsstruktur erfolgreich endet. Damit hat Werner einen artikelexkurrenten umtextadkurrent orientierten Zugriffspfad etabliert. Das allgemeine Zugriffspfadbild für diesen Pfadtyp findet sich in Abb. 3-3.

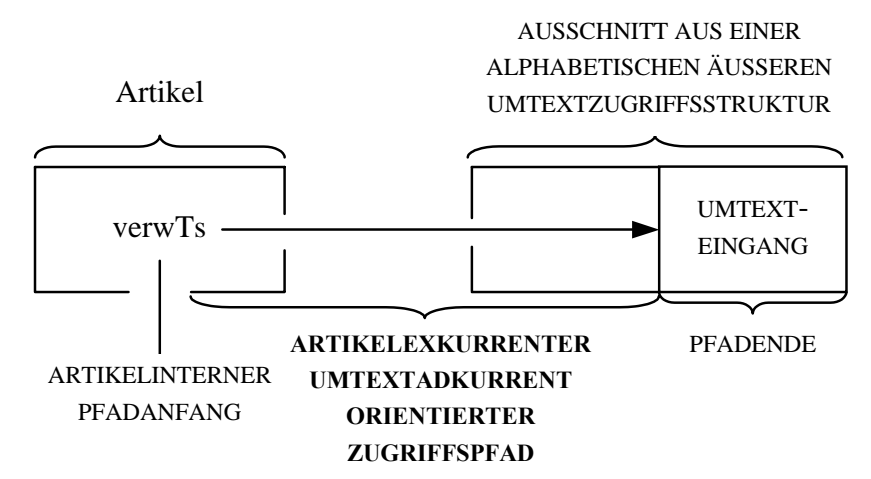

Abb. 3-3: Allgemeines Zugriffspfadbild für den Typ des artikelexkurrenten umtextadkurrent orientierten Zugriffspfades; Abkürzungen: verwTs = verweisvermittelndes Textsegment

Ein drittes Beispiel für einen mediostrukturellen binnenkurrenten äußeren Zugriffspfad ist das Folgende:

(B 3-4: Artikelexkurrenter Zugriffspfad/artikelexkurrenter artikelinkurrent orientierter Zugriffspfad) 
Werner beschäftigt sich mit Diminution. In dem Wörterbuchartikel zum Lemmazeichen Tierchen im Duden-GW findet er folgende usuelle vollständige Verweisangabe (a) "†Tier (1)“. Die abstrakte hierarchische und isomorphe konkrete hierarchische Angabemikrostruktur der Verweisangabe (a) ist in Abb. 3-4 dargestellt.

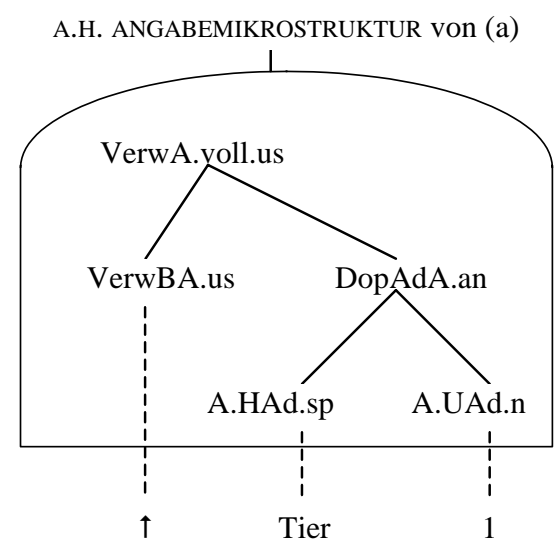

Abb. 3-4: Kommentierter Strukturgraph zur abstrakten hierarchischen (und isomorphen konkreten hierarchischen) Angabemikrostruktur der Verweisangabe (a). Abkürzungen: A. = abstrakt; H. = hierarchisch; VerwA.voll.us $=$ usuelle vollständige Verweisangabe; VerwBA.us. = usuelle Verweisbeziehungsangabe; DopAdA.an = alphanumerische Doppeladressenangabe; A.HAd.sp = sprachliche Hauptadressenangabe; A.UAd. $n=$ numerische Unteradressenangabe; ",X-Y" bedeutet (von unten nach oben gelesen) so viel wie $X$ ist eine Teilangabe von $Y$; , $\mathrm{U} \cdots \cdot . \cdot \mathrm{V}^{\prime \prime}$ bedeutet so viel wie $U \in V$ (U ist ein Element von $V$ )

Wird anhand von verweisvermittelnden Textsegmenten, mit denen eine Doppeladresse genannt wird, ein Verweis erschlossen und schließt sich eine erfolgreiche Verweisbefolgungshandlung an, wird stets ein mediostruktureller $\mathrm{Zu}$ griffspfad etabliert, der eine inkurrente mediostrukturelle Orientierung aufweist, so dass er in einen akzessiven Eintrag oder in einem anderen Zugriffssektor (vgl. 3.3) hineinführt. Die inkurrent orientierten Zugriffspfade werden — obwohl ihr Pfadende innerhalb akzessiver Einträge liegt — zu den äußeren Zugriffspfaden gerechnet, da sie stets über äußere Zugriffstextelemente führen.

In B 3-4 erschließt Werner anhand der artikelorientierten Verweisangabe einen artikelorientierten Verweis. Diesem folgt er dadurch, dass er unter Zuhilfenahme der Doppeladresse |Tier 1| als mediostrukturellem Leitelement eine externe Verweisbefolgungshandlung dadurch ausführt, dass er eine externe Zugriffshandlung ausführt. Diese ist erfolgreich, und Werner kommt zunächst zum Lemma des folgenden Wörterbuchartikels:

wa3: Tier [...], das; -[e]s, -e [...] 1. mit Sinnes- und Atmungsorganen ausge- 
stattetes, sich von anderen tierischen od. pflanzlichen Organismen ernährendes, in der Regel frei bewegliches Lebewesen [...]

Nach Erreichung des Lemmas „Tier“ führt Werner unter Zuhilfenahme der Unteradresse $|1|$ eine interne Zugriffshandlung aus, die ihn zum Pfadende, der Polysemieangabe „1.“, bringt. Damit hat Werner einen artikelexkurrenten artikelinkurrent orientierten Zugriffspfad etabliert. Das allgemeine Zugriffspfadbild für den zugehörigen Zugriffspfadtyp findet sich in Abb. 3-5.

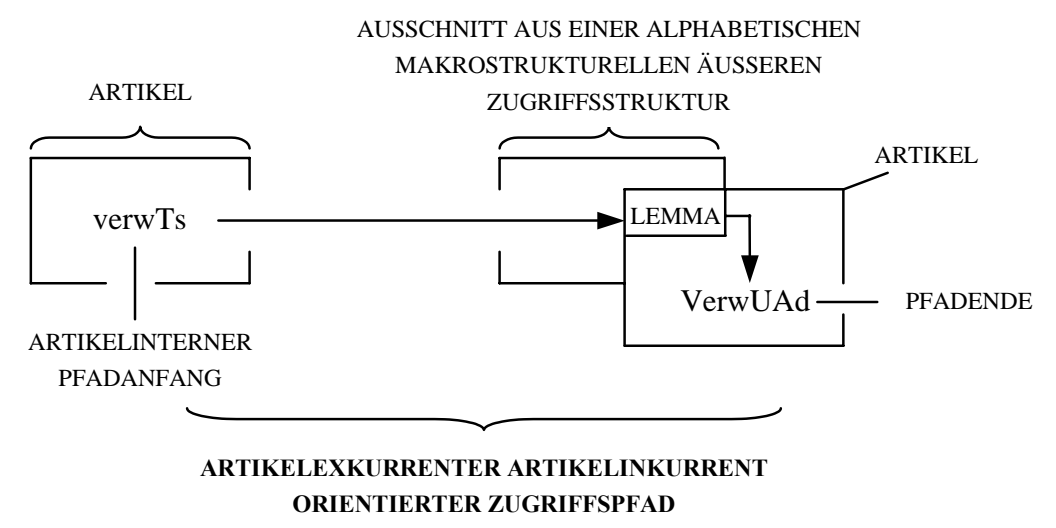

Abb. 3-5: Allgemeines Zugriffspfadbild für den Typ des artikelexkurrenten artikelinkurrent orientierten Zugriffspfades; Abkürzungen: verwTs = verweisvermittelndes Textsegment; VerwUAd = Verweisunteradresse

Um über die bereits erwähnte typologische Vielfalt, die bei den mediostrukturellen binnenkurrenten äußeren Zugriffspfaden gegeben ist, einen Überblick zu gewinnen, werden im Folgenden die wichtigsten Typologieausschnitte betrachtet. Mit dem Typologiekriterium „Lage des außenorientierten Pfadanfangs" bilden wir zunächst einen Typologieausschnitt unter dem Aspekt, zu welchem Typ von akzessiven Einträgen welcher Typ von Zugriffspfaden gehört ohne Rücksicht auf die mediostrukturelle Orientierung; dieser findet sich - eingebettet in seine typologische Umgebung — in Abb. 3-6.

Von den Definitionen, die zu dem Typologieausschnitt in Abb. 3-6 gehören, seien die beiden folgenden genannt:

(D 3-5: exkurrenter Zugriffspfad)

Ein exkurrenter Zugriffspfad ist ein mediostruktureller binnenkurrenter äußerer Zugriffspfad, dessen Pfadanfang in einem akzessiven Eintrag oder einer Textverbundkonstituente liegt, in dem sein wörterbuchinternes Pfadende nicht situiert ist. 
(D 3-6: artikelexkurrenter Zugriffspfad)

Ein artikelexkurrenter Zugriffspfad ist ein mediostruktureller binnenkurrenter äußerer Zugriffspfad, dessen Pfadende nicht im gleichen Wörterbuchartikel liegt wie sein Pfadanfang.

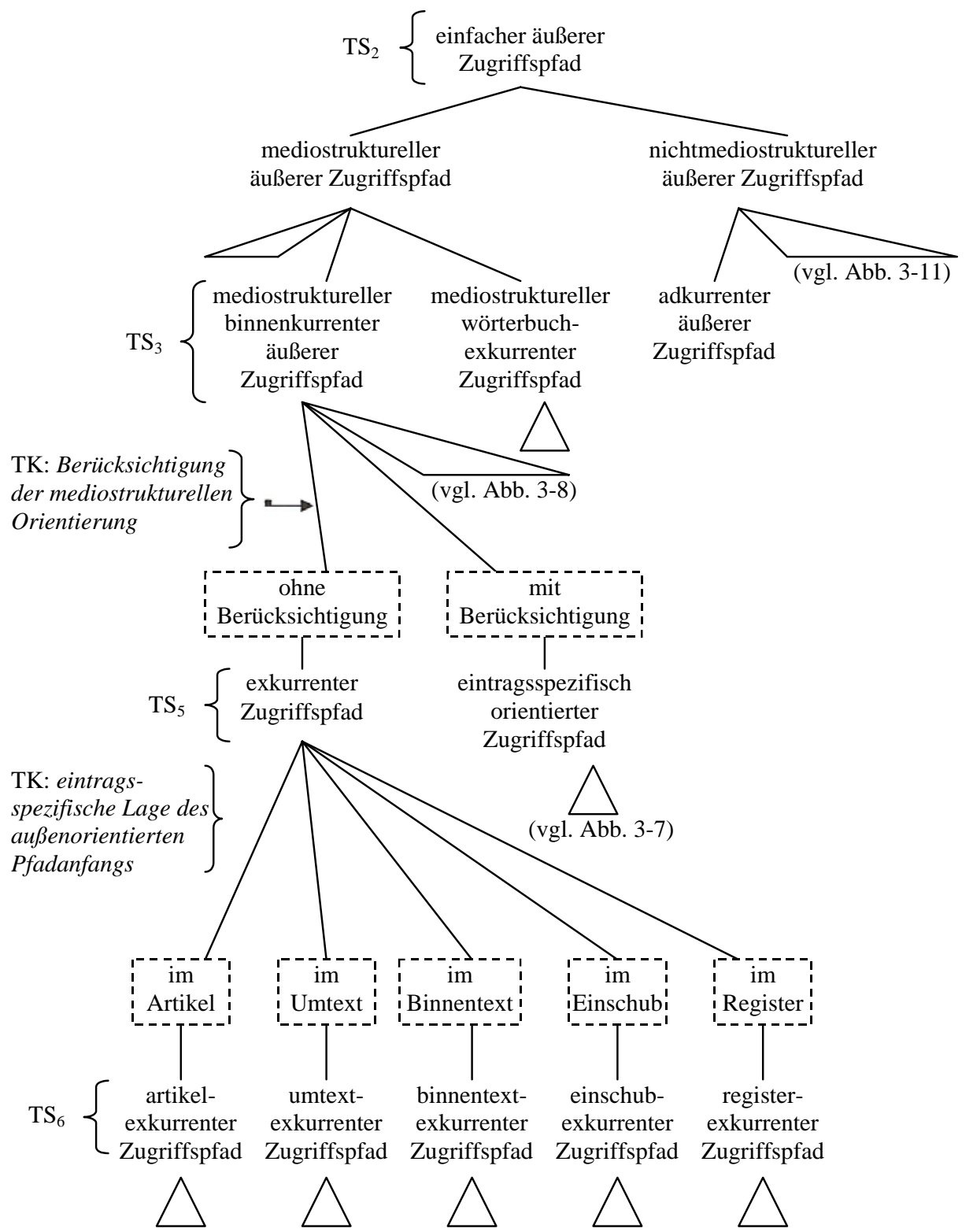

Abb. 3-6: Zweiter partiell erweitert kommentierter Typologiegraph zu einem Ausschnitt aus einer Typologie von Zugriffspfaden 
Die Frage, wozu ein Zugriffspfadtyp nützlich ist, bei dessen Bildung die mediostrukturelle Orientierung nicht berücksichtigt wird, ist berechtigt; sie wird aber wahrscheinlich nur von jemand gestellt, der sich noch nicht vor die Aufgabe gestellt sah, das qualitativ-quantitative mediostrukturelle Profil z.B. eines Lern- oder Konsultationswörterbuchs (sensu Wiegand 2006; vgl. Schierholz und Wiegand 2005) zu berechnen. Dann muss er nämlich z.B. angeben können, wie viele pfadanfangsverschiedene exkurrente Zugriffspfadtypen gegeben sind, wie viel Prozent davon z.B. artikelexkurrent und wie viel Prozent davon umtextexkurrent sind.

Beim nächsten Typologieausschnitt in Abb. 3-7 wird die mediostrukturelle Orientierung der mediostrukturellen binnenkurrenten äußeren Zugriffspfade berücksichtigt. In den Termini für Zugriffspfadtypen tritt dann stets eine Form von orientiert auf.

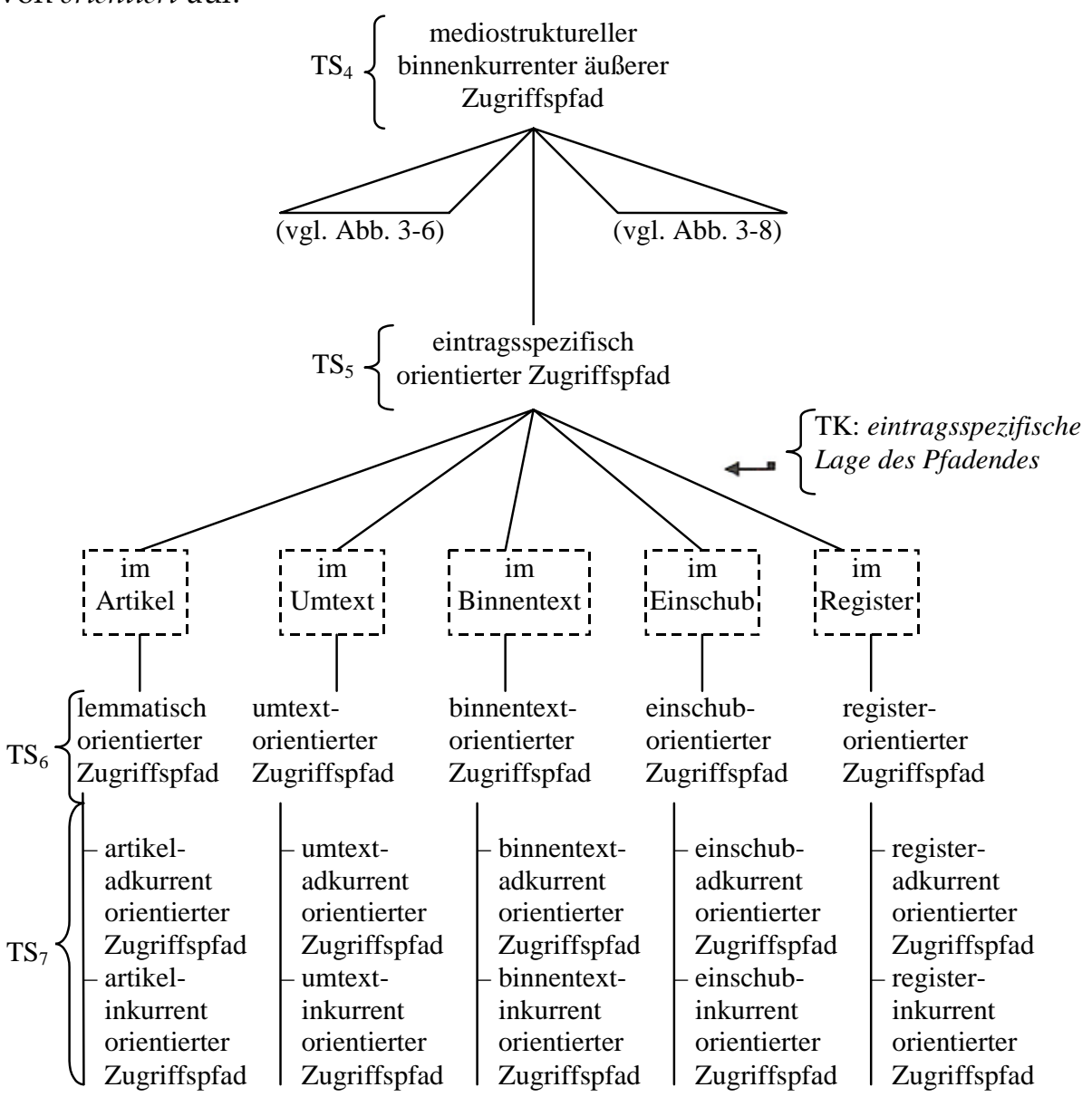

Abb. 3-7: Dritter partiell erweitert kommentierter Typologiegraph zu einem Ausschnitt aus einer Typologie von Zugriffspfaden 
Von den Definitionen, die zu dem Typologieausschnitt in Abb. 3-7 gehören, sei die Folgende genannt:

(D 3-7: umtextorientierter Zugriffspfad)

Ein umtextorientierter Zugriffspfad ist ein mediostruktureller binnenkurrenter äußerer Zugriffspfad, dessen Pfadende in einem Umtext liegt.

Es ist klar, dass für alle Zugriffspfadtypen, deren Pfadende ein Element einer äußeren Zugriffsstruktur ist, ein gemeinsamer Obertyp gebildet werden kann, nämlich der Typ des adkurrent orientierten Zugriffspfades, der wie folgt definiert ist:

(D 3-8: adkurrent orientierter Zugriffspfad)

Ein adkurrent orientierter Zugriffspfad ist ein mediostruktureller binnenkurrenter äußerer Zugriffspfad, dessen Pfadende ein Element einer äußeren Zugriffsstruktur ist.

Weiterhin ist wohl deutlich, dass auch für alle Zugriffspfadtypen, deren Pfadende nicht in einer äußeren Zugriffsstruktur liegt, aber nur über ein äußeres Zugriffstextelement erreichbar ist, ein Obertyp gebildet werden kann, nämlich der Typ des inkurrent orientierten Zugriffspfades, der wie folgt definiert ist:

(D 3-9: inkurrent orientierter Zugriffspfad)

Ein inkurrent orientierter Zugriffspfad ist ein mediostruktureller binnenkurrenter äußerer Zugriffspfad, dessen Pfadende innerhalb eines akzessiven Eintrages liegt und nur über dessen äußeres Zugriffstextelement erreichbar ist.

Entsprechend ergibt sich der Typologieausschnitt in Abb. 3-8.

Von den Definitionen, die zu dem Typologieausschnitt in Abb. 3-8 gehören, seien die beiden folgenden genannt:

(D 3-10: binnentextadkurrent orientierter Zugriffspfad)

Ein binnentextadkurrent orientierter Zugriffspfad ist ein mediostruktureller binnenkurrenter äußerer Zugriffspfad, dessen Pfadende ein Binnentexteingang eines akzessiven Binnentexteintrags ist.

(D 3-11: umtextinkurrent orientierter Zugriffspfad)

Ein umtextinkurrent orientierter Zugriffspfad ist ein mediostruktureller binnenkurrenter äußerer Zugriffspfad, dessen Pfadende in einem akzessiven Umtexteintrag liegt und nur über den zugehörigen Umtexteingang erreichbar ist.

Will man bei der Bildung von mediostruktrurellen Zugriffspfadtypen die Lage des Pfadanfangs, die mediostrukturelle Orientierung und die Lage des Pfad- 
endes zusammen berücksichtigen, lässt sich dies am einfachsten dadurch bewerkstelligen, dass man den Typologieausschnitt in Abb. 3-6 um weitere Untertypen erweitert, so dass sich der Typologiegraph in Abb. 3-9 auf der nächsten Seite ergibt.

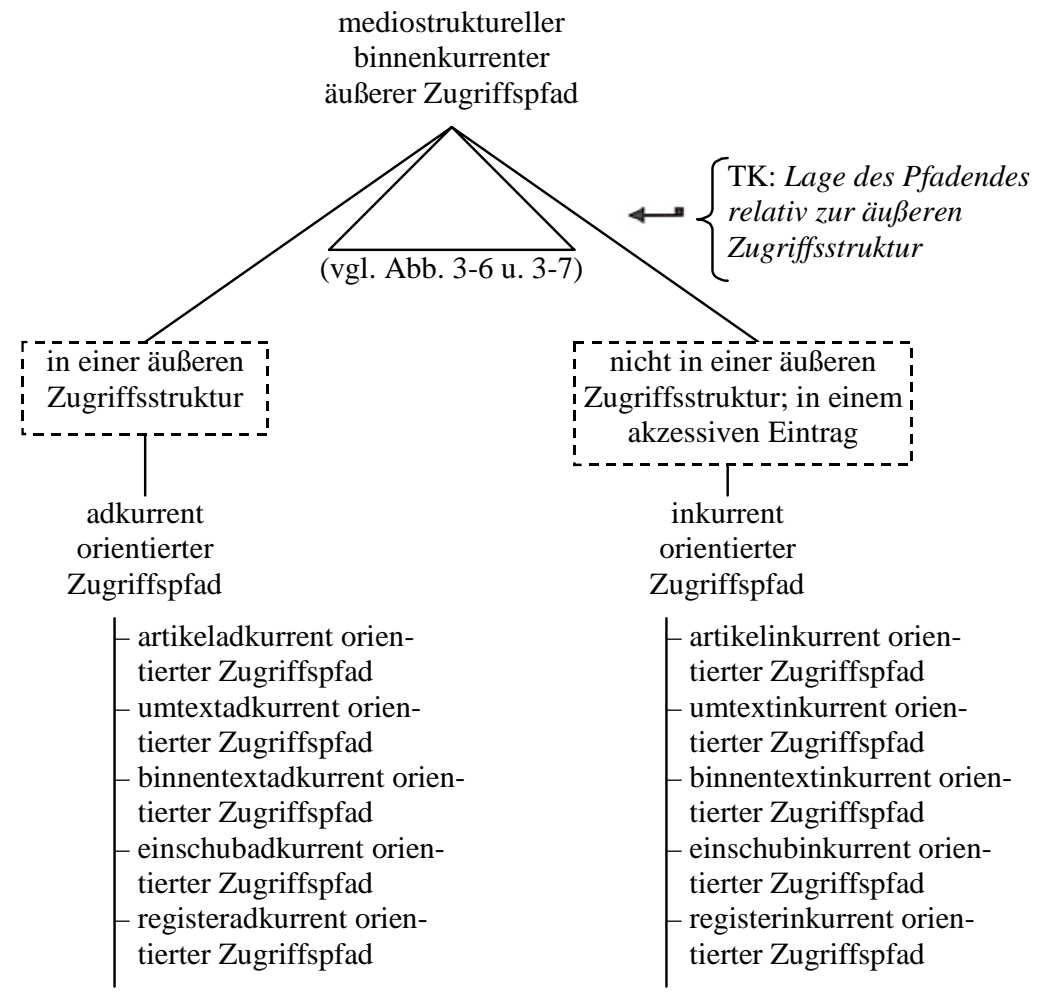

Abb. 3-8: Vierter partiell erweitert kommentierter Typologiegraph zu einem Ausschnitt aus einer Typologie von Zugriffspfaden

Von den Definitionen, die zu dem Typologieausschnitt in Abb. 3-9 gehören, seien die beiden folgenden genannt:

(D 3-12: umtextexkurrenter artikeladkurrent orientierter Zugriffspfad)

Ein umtextexkurrenter artikeladkurrent orientierter Zugriffspfad ist ein mediostruktureller binnenkurrenter äußerer Zugriffspfad, dessen Pfadanfang in einem Umtext liegt und dessen Pfadende ein Lemma ist.

(D 3-13: registerexkurrenter umtextinkurrent orientierter Zugriffspfad)

Ein registerexkurrenter umtextinkurrent orientierter Zugriffspfad ist ein mediostruktureller binnenkurrenter äußerer Zugriffspfad, dessen Pfadanfang in einem Register liegt und dessen umtextinternes Pfadende nur über einen Umtexteingang erreichbar ist. 


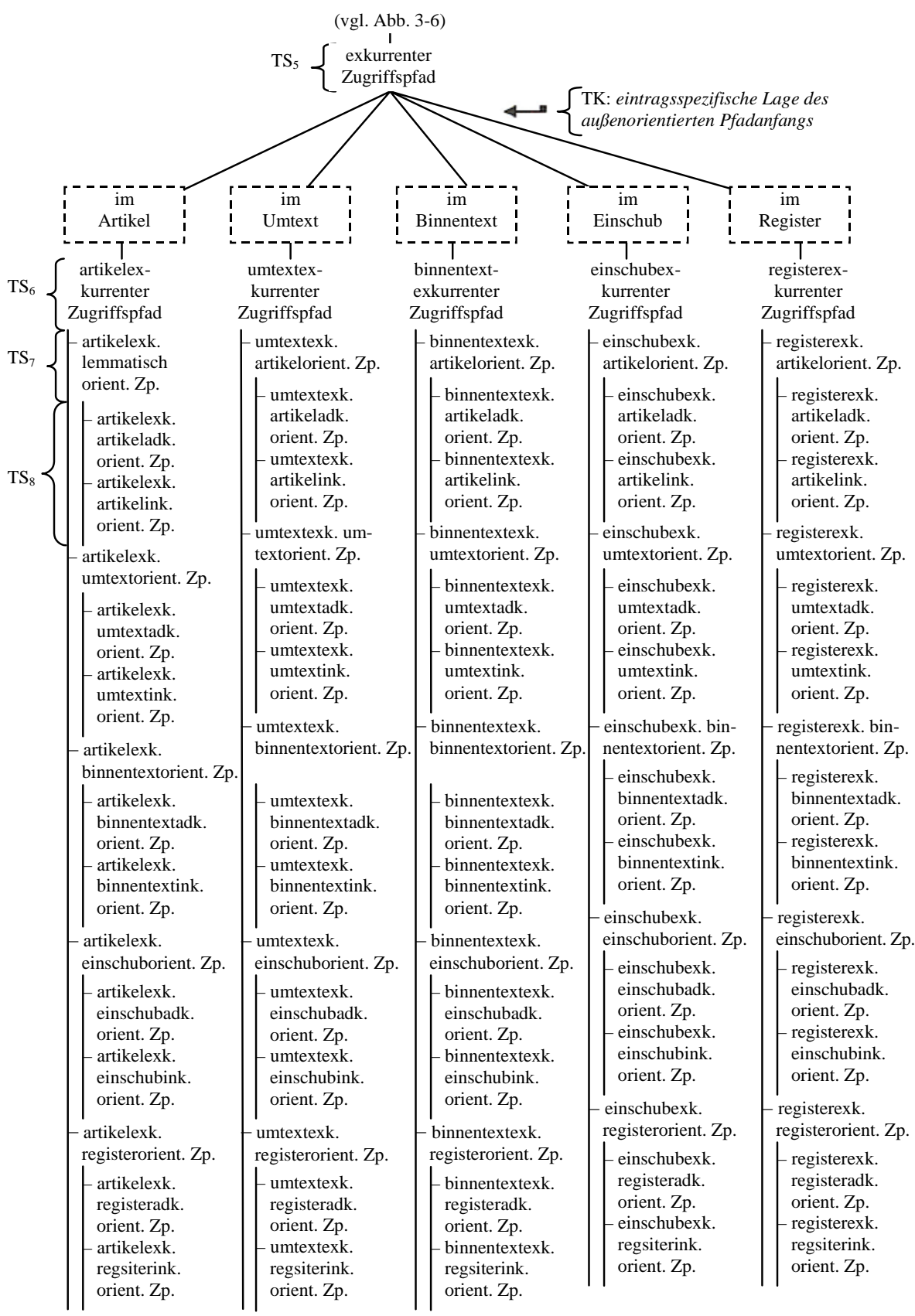

Abb. 3-9: Fünfter partiell erweitert kommentierter Typologiegraph zu einem Ausschnitt aus einer Typologie von Zugriffspfaden; Abkürzungen: exk. = exkurrent; adk. = adkurrent; ink. = inkurrent; Zp. = Zugriffspfad 


\subsection{Mediostrukturelle wörterbuchexkurrente äußere Zugriffspfade}

Auf den Typ des mediostrukturellen wörterbuchexternen äußeren Zugriffspfades kann hier (aus Platzgründen) nur sehr kurz eingegangen werden. Auch auf die Präsentation eines Typologieausschnittes muss verzichtet werden. Mit dem Pfadanfang mediostruktureller wörterbuchexkurrenter Zugriffspfade wird immer eine wörterbuchexterne Verweisadresse genannt. Solche Verweisadressen sind z.B. Quellentitel, Titel von fachlichen Publikationen oder Belegstellen. Die mit dem jeweiligen verweisvermittelnden Textsegment genannten wörterbuchexternen Adressen sind meistens verdichtet; dann müssen sie in Umtexten, wie z.B. Quellenverzeichnissen oder Literaturverzeichnissen, ergänzt werden, bevor Verweisbefolgungshandlungen ausgeführt werden. Für historische Wörterbücher beispielsweise gilt, dass sie Schnittstellenelemente aufweisen, die die Benutzer in die Lage versetzen, mediostrukturelle wörterbuchexkurrente quellenorientierte Zugriffspfade zu etablieren. Für Fachwörterbücher gilt beispielsweise, dass sie Schnittstellenelemente aufweisen sollten, um wörterbuchexkurrente fachliteraturorientierte Zugriffspfade zu etablieren.

\subsubsection{Nichtmediostrukturelle äußerer Zugriffspfade}

Bei den nichtmediostrukturellen äußeren Zugriffspfaden werden — neben den bereits in Abschnitt 3.1.1.1 behandelten adkurrenten äußeren Zugriffspfaden die nichtmediostrukturellen binnenkurrenten äußeren Zugriffspfade und die nichtmediostrukturellen wörterbuchexkurrenten Zugriffspfade unterschieden.

\subsection{Nichtmediostrukturelle binnenkurrente äußere Zugriffspfade}

Zugriffspfade, die zum Typ des nichtmediostrukturellen binnenkurrenten äußeren Zugriffspfades gehören, werden dann von Benutzern etabliert, wenn es bei der Wörterbuchbenutzung zu Textrezeptionsstörungen kommt und der Benutzer diese dadurch beheben möchte, dass er das gleiche Wörterbuch benutzt, anhand dessen die Textrezeptionsstörung entstanden ist, wie in folgendem Beispiel:

(B 3-5: Nichtmediostruktureller binnenkurrenter äußerer Zugriffspfad/artikelexkurrenter und artikeladkurrenter äußerer Zugriffspfad)

Werner liest einen englischen Text und kennt das Wort howl nicht. Er schlägt im WNED nach und findet im Artikel mit dem Lemma "howl“ folgende Bedeutungsparaphrasenangabe: "to make a loud sustained doleful sound“. In dieser Angabe kennt er "sustained" nicht. Werner führt daraufhin mit dem Leitelement |sustain| eine externe Zugriffshandlung erfolgreich aus, so dass er im WNED zum Lemma "sus'tain“ kommt. Damit hat er einen artikelexkurrenten und artikeladkurrenten Zugriffspfad etabliert. Das zugehörige allgemeine Zugriffspfadbild findet sich in Abb. 3-10. 


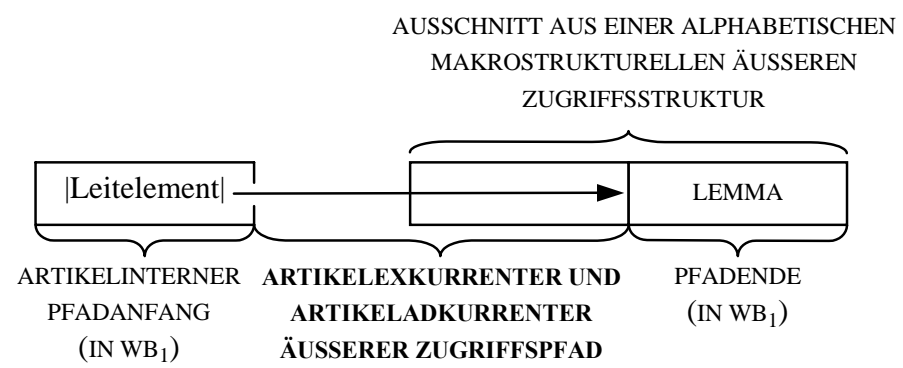

Abb. 3-10: Allgemeines Zugriffspfadbild für den Typ des artikelexkurrenten und artikeladkurrenten äußeren Zugriffspfades; Abkürzungen: $\mathrm{WB}=$ Wörterbuch

Einige wichtige Untertypen des Typs des nichtmediostrukturellen binnenkurrenten äußeren Zugriffspfades sind die in Abb. 3-11 zu einem Typologieausschnitt zusammengefasst.

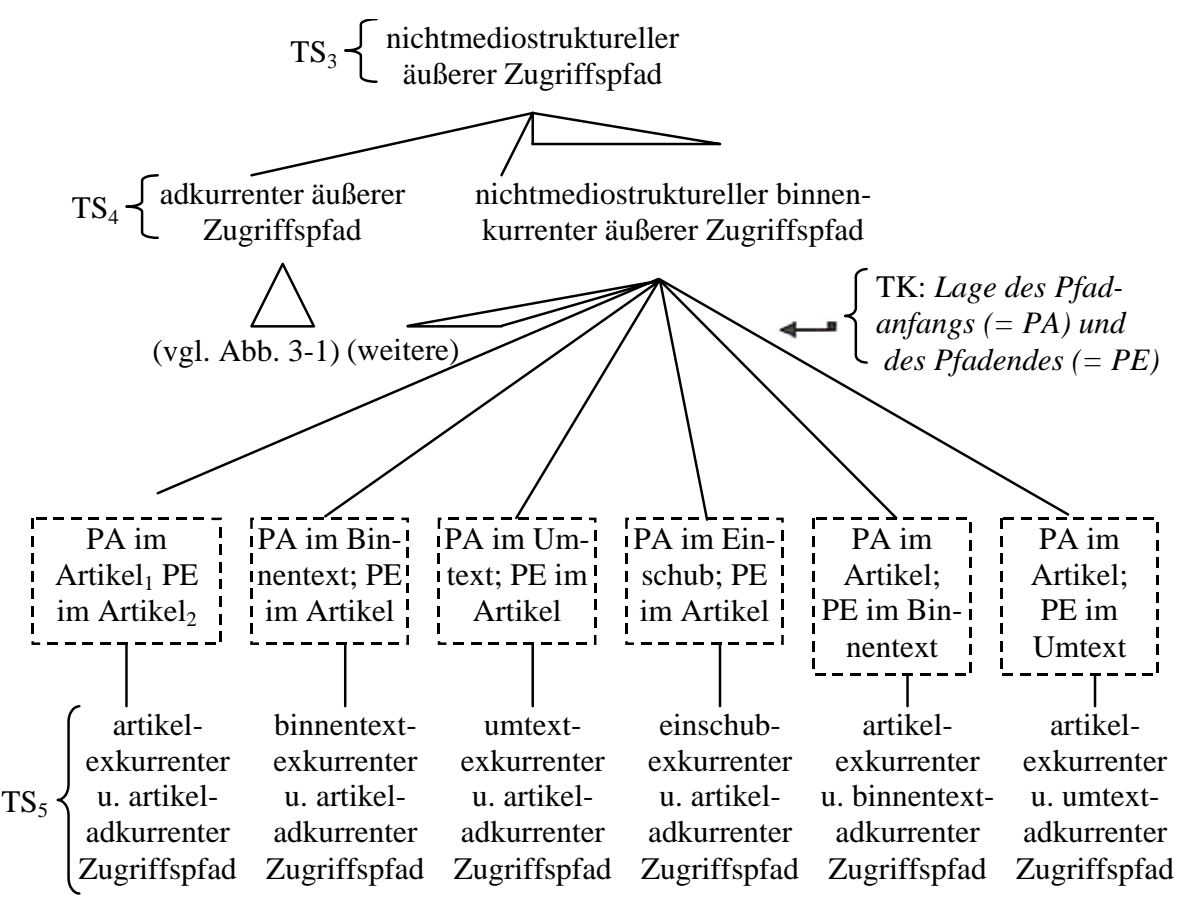

Abb. 3-11: Sechster partiell erweitert kommentierter Typologiegraph zu einem Ausschnitt aus einer Typologie von Zugriffspfaden 


\subsection{Nichtmediostrukturelle wörterbuchexkurrente äußere Zugriffspfade}

Zugriffspfade, die zum Typ des nichtmediostrukturellen wörterbuchexkurrenten äußeren Zugriffspfades gehören, werden dann von Benutzern etabliert, wenn es bei der Benutzung eines Wörterbuchs $\mathrm{Wb}_{1} \mathrm{zu}$ den Textrezeptionsstörungen kommt und der Benutzer diese dadurch beheben möchte, dass er ein anderes Wörterbuch $\mathrm{Wb}_{2}$ benutzt. Solche Situationen treten vor allem bei der Benutzung von zweisprachigen Wörterbüchern sowie bei der Benutzung von Fachwörterbüchern auf. Die nichtmediostrukturellen wörterbuchexkurrenten äußeren Zugriffspfade, die öfter etabliert werden, gehören entweder zu den artikel- und wörterbuchexkurrenten artikeladkurrenten Zugriffspfaden oder zu den artikel- und wörterbuchexkurrenten registeradkurrenten Zugriffspfaden. Da die Wörterbuchbenutzung, in deren Rahmen nichtmediostrukturelle wörterbuchexkurrente äußere Zugriffspfade etabliert werden, wohl relativ selten auftritt, wird hier auf die Präsentation eines Typologieausschnittes verzichtet.

\subsubsection{Zusammengesetzte äußere Zugriffspfade}

Gegeben sei folgendes Beispiel:

(B 3-6: Zusammengesetzter äußerer Zugriffspfad/registeradkurrenter äußerer Zugriffspfad mit binnenkurrentem artikelorientiertem Anschlusspfad)

Werner benötigt bedeutungsverwandte Wörter zu dem Verb herausfischen; sein Benutzerziel ist mithin das Auffinden solcher Wörter. Er greift zum Dornseiff (2004) und führt mit dem Leitelement |herausfischen| eine externe Registerzugriffshandlung erfolgreich aus, die ihn zu dem Registereingang "herausfischen" des einfachen akzessiven Registereintrags: „herausfischen 20.8 Aneignen" führt. Damit hat Werner einen registeradkurrenten äußeren Zugriffspfad etabliert; um sein Benutzerziel zu erreichen, muss er sodann anhand der einfachen Registerangabe "20.8 Aneignen", die wie eine reduzierte Verweisangabe funktioniert, das mediostrukturelle Leitelement |20.8 Aneignen| erschließen und eine externe Verweisbefolgungshandlung ausführen, die ihn zu dem Sachgruppenartikel „20.8 Aneignen" führt. Nach dem Zugriff auf diesen Artikeltitel als Eingang zum sachgruppenbezogenen Wörterbuchartikel hat Werner einen binnenkurrenten artikelorientierten Anschlusspfad etabliert, dessen Pfadende der Artikeltitel ist, und damit insgesamt einen zusammengesetzten äußeren Zugriffspfad, der zum Typ des registeradkurrenten äußeren Zugriffspfades mit binnenkurrentem artikelorientiertem Anschlusspfad gehört. Das Kriterium dafür, dass im Beispiel B 3-6 nicht zwei unabhängige Zugriffspfade vorliegen, sondern nur ein zusammengesetzter äußerer Zugriffspfad, besteht darin, dass ein Benutzerziel alle pfadetablierenden Benutzungshandlungen determiniert. 
Der Typ des zusammengesetzten äußeren Zugriffspfades lässt sich wie folgt definieren:

(D 3-14: zusammengesetzter äußerer Zugriffspfad)

Ein zusammengesetzter äußerer Zugriffspfad ist ein äußerer Zugriffspfad, der aus einen adkurrenten äußeren Zugriffspfad als Anfangspfad besteht, an dem sich ein mediostruktureller binnenkurrenter äußerer Zugriffspfad anschließt, wobei die Bedingung gilt, dass alle pfadetabliertenden Benutzungshandlungen von nur einem Benutzerziel determiniert sind.

Das allgemeine Zugriffspfadbild für den Typ des registeradkurrenten äußeren Zugriffspfades mit binnenkurrentem artikelorientiertem Anschlusspfad findet sich in Abb. 3-12.

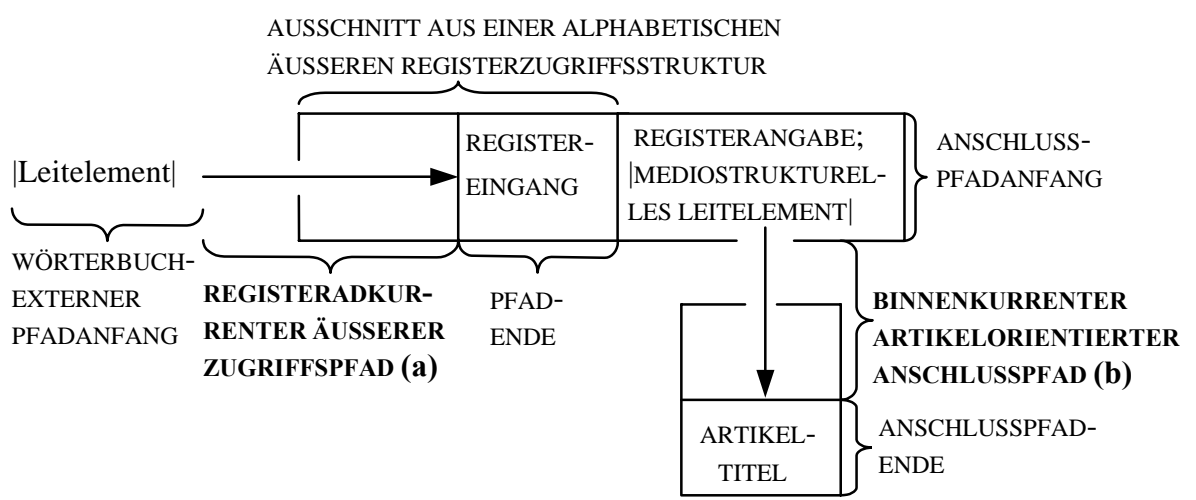

(a) + (b) = ZUSAMMENGESETZTER ÄUSSERER ZUGRIFFSPFAD

Abb. 3-12: Allgemeines Zugriffspfadbild für den Typ des registeradkurrenten äußeren Zugriffspfades mit binnenkurrentem artikelorientiertem Anschlusspfad

Die meisten zusammengesetzten äußeren Zugriffspfade, die bei der Wörterbuchbenutzung etabliert werden, gehören zum Typ des registeradkurrenten äußeren Zugriffspfades mit binnenkurrentem artikelorientiertem Anschlusspfad.

Bei der Benutzung von Spezialwörterbüchern sowie allgemeinsprachlichen Wörterbüchern mit reichhaltiger Umtextausstattung werden jedoch auch Zugriffspfade etabliert, die zu den anderen Typen gehören. Daher wird in Abb. 3-13 ein kleiner Ausschnitt aus einer Typologie von zusammengesetzten Zugriffspfaden präsentiert. 


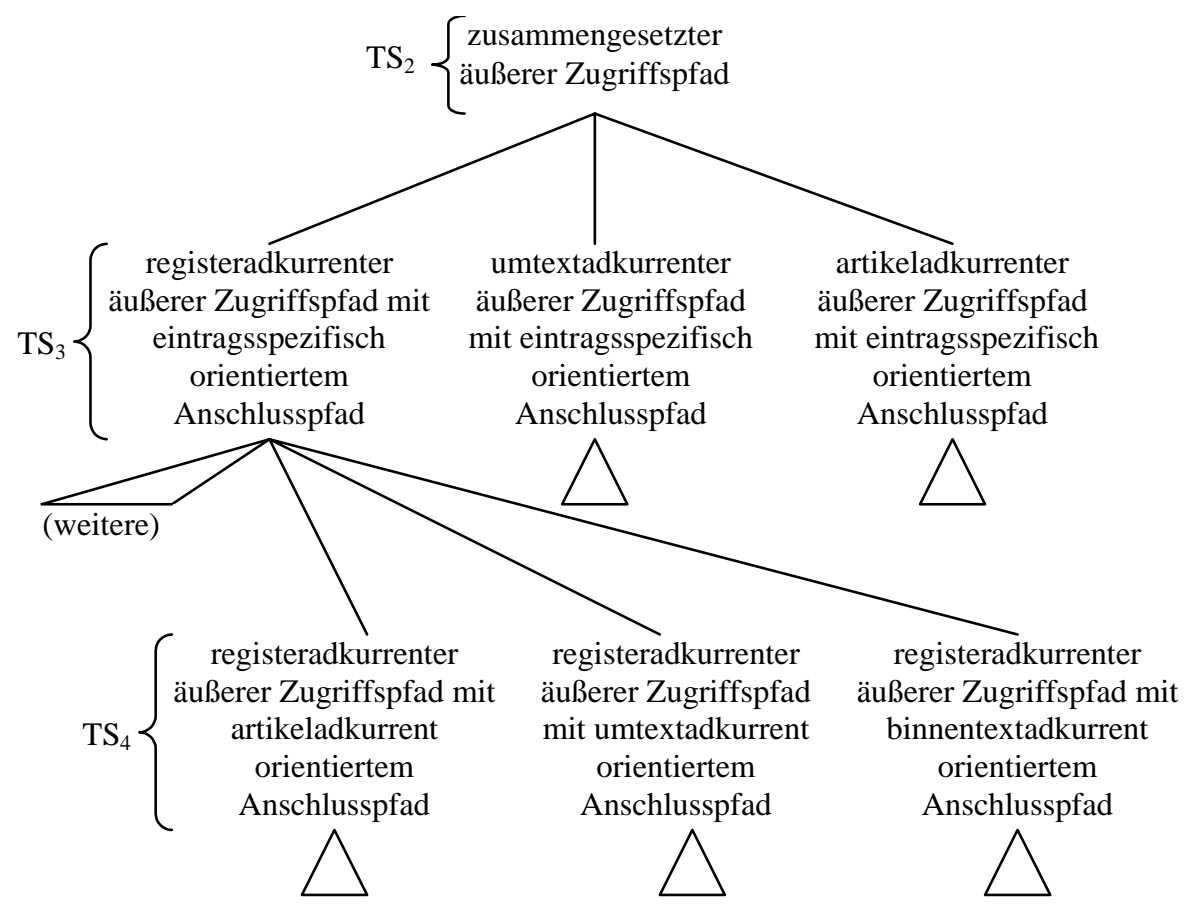

Abb. 3-13: Siebenter nichtkommentierter Typologiegraph zu einem Ausschnitt aus einer Typologie von Zugriffspfaden

Zum Schluss dieses Abschnittes sei wenigstens erwähnt, dass für die Lern- und Konsultationswörterbücher (sensu Schierholz und Wiegand 2005 und Wiegand 2006a), zu den z.B. die „Wörterbücher zur Sprach- und Kommunikationswissenschaft" [WSK] gehören, wenn diese zur wörterbuchinternen Fachwissensrecherche (sensu Wiegand 2006a) benutzt werden, vom Fachlexikographen empfohlene Zugriffspfadfolgen angegeben werden können, die von zusammengesetzten äußeren Zugriffspfaden unterschieden werden müssen, da sie nicht von einem Benutzerziel determiniert werden, sondern durch verweismotivierte Konsultationshandlungen (sensu Wiegand 1998: 408ff) verknüpft sind. Zugriffspfadfolgen lassen sich z.B. wie folgt angeben:

Zugriffspfadfolge I:

(1) Umtextexkurrenterse synopseartikeladkurrent orientierter äußerer Zugriffspfad

(2) Synopseartikelexkurrenter einzelartikeladkurrent orientierter äußerer Zugriffspfad

Zugriffspfadfolge II:

(1) Einzelartikeladkurrenter äußerer Zugriffspfad 
(2) Einzelartikelexkurrenter synopseartikeladkurrent orientierter äußerer Zugriffspfad

(3) Synopseartikelexkurrenter umtextadkurrent $t_{\mathrm{SE}}$ orientierter äußerer Zugriffspfad

Der untere Index "SE" besagt, dass es sich bei dem Umtext um die Systematische Einführung zu einem WSK-Band handelt, der nach Paragraphen gegliedert ist, an deren Ende jeweils eine feste Verweisposition situiert ist, in der auf die Synopseartikel verwiesen wird.

\subsection{Akzessive Angaben und äußere Zugriffspfade}

Im Folgenden soll gezeigt werden, dass und wie alle Typen von akzessiven Angaben, die zum Typologieausschnitt in Abb. 2-1 gehören, mit der Terminologie für äußere Zugriffspfade in einheitlicher Weise definiert werden können. Allerdings können (aus Platzgründen) nur einige Definitionen angegeben werden. Begonnen sei mit der Definition des Typs der extern akzessiven Angabe.

(D 3-15: extern akzessive Angabe)

Eine extern akzessive Angabe ist eine akzessive Angabe, die entweder über adkurrente äußere Zugriffspfade oder über registeradkurrente äußere Zugriffspfade mit binnenkurrentem eintragsspezifisch orientiertem Anschlusspfad erreichbar ist.

Die drei in Abb. 2-1 angegebenen Untertypen des Typs der extern akzessiven Angabe lassen sich entsprechend wie folgt definieren:

(D 3-16: extern direkt akzessive Angabe)

Eine extern direkt akzessive Angabe ist eine akzessive Angabe, die nur über adkurrente äußere Zugriffspfade erreichbar ist.

(D 3-17: extern indirekt akzessive Angabe)

Eine extern indirekt akzessive Angabe ist eine akzessive Angabe, die nur über registeradkurrente äußere Zugriffspfade mit binnenkurrentem eintragsspezifisch orientiertem Anschlusspfad erreichbar ist.

(D 3-18: extern direkt und indirekt akzessive Angabe)

Eine extern direkt und indirekt akzessive Angabe ist eine Angabe, die sowohl über adkurrente äußere Zugriffspfade als auch über registeradkurrente äußere Zugriffspfade mit binnenkurrentem eintragsspezifisch orientiertem Anschlusspfad erreichbar ist.

Es ist klar, dass man bei den Definitionen von akzessiven Angaben nicht notwendigerweise auf die Terminologie für die Zugriffspfadtypen zurückgreifen 
muss; vielmehr lassen sich die akzessiven Angaben auch unter Rückgriff auf die Typen von äußeren Zugriffsstrukturen sowie einige Typen von Benutzungshandlungen definieren. Solche Definitionen sind aber komplexer.

\subsection{Verzeichnisexkurrente äußere Zugriffspfade}

Es gibt Wörterbücher mit lexikographischem Inhaltsverzeichnis - wie z.B. Duden-6${ }^{6}$ DUW - , und es gibt Wörterbücher ohne lexikographisches Inhaltsverzeichnis - wie z.B. Duden- ${ }^{4}$ GFWB. Insbesondere bei den Ersteren fungiert die Paginierungsreihe als numerische äußere Zugriffsstruktur; sie heißt Seitenzahlzugriffsstruktur. Ihr direkter Zugriffsbereich ist die Menge aller paginierten Wörterbuchseiten. $\mathrm{Zu}$ jeder Seitenzahl als einem numerischen äußeren $\mathrm{Zu}$ griffstextelement gehört als Zugriffssektor eine Wörterbuchseite. Die Seitenzahlzugriffsstruktur ist eine mediostrukturelle äußere Zugriffsstruktur, so dass auf deren Zugriffstextelemente nur mit Hilfe mediostruktureller Leitelemente zugegriffen werden kann. Der erste Teil des lexikographischen Inhaltsverzeichnisses aus Steinhauer (2005) hat folgende Form:

\begin{tabular}{|c|c|}
\hline \multicolumn{2}{|l|}{ 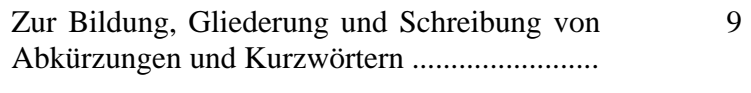 } \\
\hline Im Register verwendete Kürzel ........................... & 14 \\
\hline Verzeichnis der Abkürzungen und Kurzwörter & 15 \\
\hline \multicolumn{2}{|l|}{ Abkürzungslisten: } \\
\hline Deutsche Bundesländer (offizielle & \\
\hline 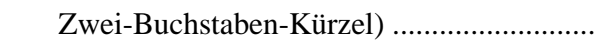 & 82 \\
\hline 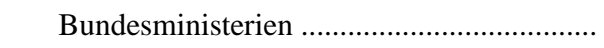 & 82 \\
\hline 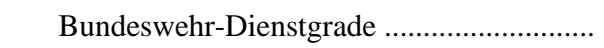 & 83 \\
\hline 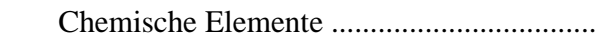 & 93 \\
\hline 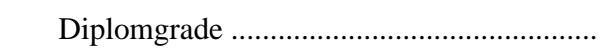 & 115 \\
\hline 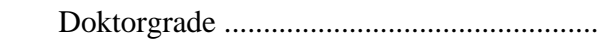 & 122 \\
\hline IATA-Codes deutscher Flughäfen .............. & 163 \\
\hline
\end{tabular}

Ganz allgemein gilt, dass lexikographische Inhaltsverzeichnisse - wie auch die Register - als mediostrukturelle Komponenten eines Wörterbuchs zu gelten haben. Ein lexikographisches Inhaltsverzeichnis lässt sich am besten als eine geordnete Liste von reduzierten alphanumerischen Doppeladressenangaben verstehen. Die Reduzierung besteht darin, dass keine Verweisbeziehungsangabe existiert. Jede Inhaltsverzeichniszeile, wie z.B. „Bundeswehr-Dienstgrade .......... 83" besteht aus einer sprachlichen Unteradressenangabe (hier: "Bundeswehr-Dienstgrade") und einer numerischen Hauptadressenangabe (hier: die Seitenzahl ",83“). Mit der Unteradressenangabe werden meistens Umtexttitel oder Titel von eingelagerten Binnentexten genannt. Die Ordnung 
der Liste wird durch die genannten Hauptadressen, also durch die Seitenzahlen, bestimmt: Geordnet wird nach steigenden Seitenzahlen $(9,14,15, \ldots$ usw.). Ein lexikographisches Inhaltsverzeichnis liefert also dem Benutzer - wie ein Register - Fundortdaten, die als mediostrukturelle Leitelemente fungieren können. Im Unterschied zu einem Register weist ein lexikographisches Inhaltsverzeichnis aber keine äußere Zugriffsstruktur auf: Auf ein Inhaltsverzeichnis greift man nicht - unter Zuhilfenahme von vorgegebenen Ordnungsgesetzlichkeiten $-\mathrm{zu}$, sondern man liest in ihm und kann gegebenenfalls auf eine Seite zugreifen, weil man eine intuitive Kenntnis der Ordnungsgesetztlichkeiten einer Zahlenreihe hat. Ein Benutzer, der dies tut, erbringt allerdings die gleiche oder zumindest eine sehr ähnliche kognitive Leistung wie ein Benutzer, der anhand eines alphanumerischen verweisvermittelnden Textsegmentes einen Verweis erschließt und diesem folgt. Es trägt daher zu einem vertieften systematischen Verständnis bei, wenn man ein lexikographisches Inhaltsverzeichnis als eine geordnete Menge von verweisvermittelnden Textsegmenten versteht. Man kann dann wie folgt argumentieren: Ein Benutzer-in-actu, der eine Verzeichniszeile liest, wie z.B. die Zeile „Diplomgrade $115^{\prime \prime}$, und dann zur Seite 115 kommt, erschließt zuerst anhand der Verzeichniszeile, die als verweisvermittelndes Textsegment fungiert, einen Verweis, dem er daraufhin durch die Ausführung einer externen Verweisbefolgungshandlung folgt; dies geschieht dadurch, dass er eine externe Zugriffshandlung ausführt, die erfolgreich endet mit dem Zugriff auf die Seitenzahl „115“, die ein Element der Seitenzahlzugriffsstruktur ist; es folgt eine interne Zugriffshandlung, die bei „Diplomgrade" erfolgreich endet. Ein Benutzer, der auf diese Weise z.B. vom Inhaltsverzeichnis zu einem Binnentexttitel gelangt, hat damit einen verzeichnisexkurrenten binnentextadkurrent orientierten äußeren Zugriffspfad etabliert; hinsichtlich der Wörterbuchseite als dem Zugriffssektor der Seitenzahl ist der etablierte Zugriffspfad inkurrent, nämlich wörterbuchseiteninkurrent, denn er wurde mit Hilfe einer Doppeladresse etabliert. Ein Benutzer, der vom Inhaltsverzeichnis zu einem Umtexttitel gelangt, hat einen verzeichnisexkurrenten umtextadkurrent orientierten äußeren Zugriffspfad etabliert, der ebenfalls wörterbuchseiteninkurrent ist. Die verzeichnisexkurrenten äußeren $\mathrm{Zu}$ griffspfade gehören zu den mediostrukturellen binnenkurrenten äußeren $\mathrm{Zu}$ griffspfaden. Für die, welche lexikographische Inhaltsverzeichnisse als Umtexte eines besonderen Typs begreifen, sind verzeichnisexkurrente äußere $\mathrm{Zu}$ griffspfade zugleich umtextexkurrente äußere Zugriffspfade.

\subsection{Zugriffsumwege}

In manchen Wörterbüchern sind die Schnittstellen von Benutzungshandlungen und Wörterbuchform nicht optimal gestaltet, sondern so, dass der Benutzer Zugriffsumwege in Kauf nehmen muss. Es gibt verschiedene Typen von Zugriffsumwegen, worauf ich hier nicht eingehen kann. Im Folgenden wird 
nur ein Beispiel behandelt. Wir können hierzu auf den Binnentextabschnitt aus ${ }^{2}$ Herders Sprachbuch im Beispiel B 3-2 zurückgreifen. An dessen Schluss finden sich die beiden nichtusuellen binnentextorientierten Verweisangaben ,$\square$ Wortschatz" und , $\square$ Wortbildung". In den Benutzungshinweisen mit dem Titel „Zum Aufbau des Sprachbuchs" (S. VII-VIII) findet sich folgendes Textstück:

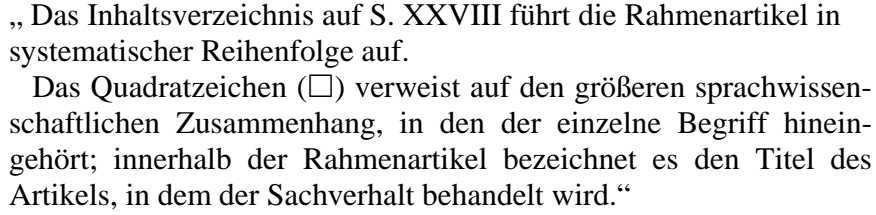

Bei den Rahmenartikeln handelt es sich um 36 eingelagerte Binnentexte ohne äußere Binnentextzugriffsstruktur. Die beiden oben genannten nichtusuellen Verweisangaben stehen im Binnentext mit dem Binnentexttitel „Fach- und Sondersprachen". Mit den beiden Verweisadressenangaben "Wortbildung" und „Wortschatz" werden Binnentexttitel genannt. Ein Benutzer, der auf diese zugreifen möchte, hat keine direkte Zugriffsmöglichkeit. Vielmehr muss er erst im Verzeichnis der Rahmenartikel im Wörterbuchvorspann nachschlagen und dort die Seitenzahl für die Seite suchen, auf der der Binnentexttitel steht und kommt erst dann auf diese Seite; der Benutzer muss also zuerst einen binnentextexkurrenten verzeichnisadkurrent orientierten äußeren Zugriffspfad etablieren und daraufhin als Anschlusspfad einen verzeichnisexkurrenten binnentextadkurrent orientierten äußeren Zugriffspfad. Eine Schnittstellengestaltung, die dem Benutzer einen Zugriffsumweg über das Verzeichnis in Form eines zusammengesetzten äußeren Zugriffspfades zumutet, ist aber unnötig und damit unprofessionell. Denn die Verweisangaben könnten einfach lauten "> Wortbildung, S. 328" und "> Wortschatz, S. 697". Oder aber man benutzt die alphabetische makrostrukturelle äußere Zugriffsstruktur auch als äußere Zugriffsstruktur für Binnentexte und ordnet die Binnentexttitel alphabetisch ein.

\subsection{Innere Zugriffspfade}

Während äußere Zugriffspfade immer über äußere Zugriffsstrukturen führen, führen innere Zugriffspfade immer über innere Zugriffsstrukturen. Außer den alphabetischen inneren Zugriffsstrukturen, die sich z.B. als positionsspezifische Zugriffsstrukturen in der Literaturposition von Fachwörterbuchartikeln finden, unterscheiden sich alle inneren Zugriffsstrukturen grundsätzlich von äußeren Zugriffsstrukturen, denn sie basieren auf dem Wissen von Benutzern über Artikelstrukturen (vgl. Wiegand 2005: 225ff). Benutzer können interne Zugriffshandlungen nur ausführen, wenn sie kognitiv über ihre individuelle interne Zugriffsstruktur verfügen. Der Pfadanfang von nichtmediostrukturellen artikelinternen Zugriffspfaden ist meistens die jeweilige lemmatische Angabe und das Pfadende die gesuchte Angabe. Bei erfahrenden Benutzern führen die 
inneren Zugriffspfade meistens über die inneren Schnellzugriffsstrukturen, weil sich die Benutzer deren Elemente gut merken können. Der Pfadanfang von mediostrukturellen inneren Zugriffspfaden ist ein verweisvermittelndes Textsegment, mit dem eine Verweisbinnenadresse genannt wird. Im Unterschied zu den eintragsübergreifenden Zugriffspfaden, die binnenkurrent verlaufen, ist der Pfadverlauf bei inneren Zugriffspfaden z.B. artikel- oder umtextintern. Eine Typologie von inneren Zugriffsstrukturen ist relativ unergiebig.

\section{6 Übersicht über die Typologie von Zugriffspfaden in Printwörter- büchern}

In der Abb. 3-14 findet sich eine Übersicht über die Typologie von Zugriffspfaden:

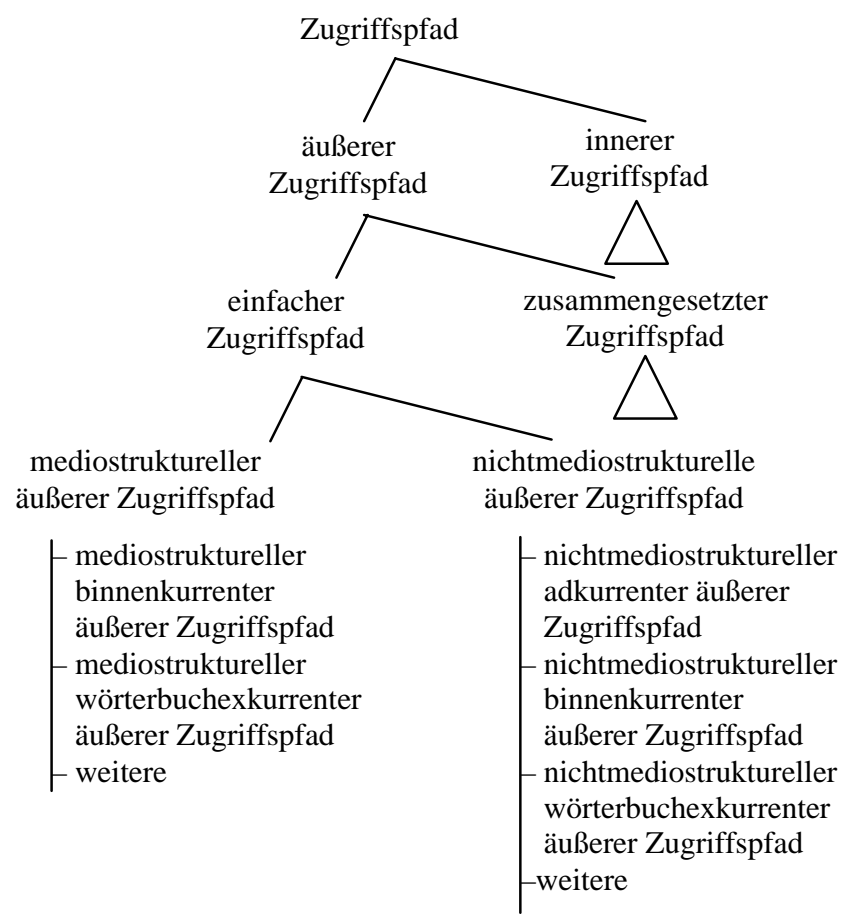

Abb. 3-14: Achter nicht kommentierter Typologiegraph: Übersicht zur Typologie von Zugriffspfaden

\subsection{Schlussbemerkung}

Die in diesem Beitrag erstmals in einer einheitlichen Perspektive zusammenhängend vorgestellte Terminologie für Zugriffspfade in Printwörterbüchern und die zugehörigen lexikographietheoretischen Begrifflichkeiten leisten bei 
der Evaluation der Datenakzessivität von Printwörterbüchern und bei der Darstellung des mediostrukturellen Profils eines Wörterbuchs sowie bei dessen systematischer Planung gute Dienste. Dies wird in einem späteren Beitrag dargestellt, der an den vorliegenden anschließt und in dem eine formale Sprache für die Darstellung von Zugriffspfaden und damit für die Darstellung von so genannten Verweissystemen präsentiert wird.

\section{Danksagung}

Werner Wolski danke ich sehr für eine hilfreiche Durchsicht des Manuskripts und Christina-Doreen Reinhard für dessen Herstellung.

\section{Literatur}

DCE = 1984: Dictionary of Contemporary English. Ein umfassendes einsprachiges Wörterbuch für Schule und Hochschule. London: Longman Group Ltd.

Dornseiff, Franz. 1934. Der deutsche Wortschatz nach Sachgruppen. Berlin/Leipzig: Walter de Gruyter.

Dornseiff, Franz. 2004. Der deutsche Wortschatz nach Sachgruppen. 8., völlig neu bearb. und mit einem vollständigen alphabetischen Zugriffsregister versehene Aufl. von Uwe Quasthoff. Mit einer lexikographisch-historischen Einführung und einer ausgewählten Bibliographie zur Lexikographie und Onomasiologie von Herbert Ernst Wiegand. Berlin/New York: Walter de Gruyter.

Duden-6 ${ }^{6}$ UW = Dudenredaktion (Hrsg.). 2007. Deutsches Universalwörterbuch. 6., überarb. und erw. Aufl. Mannheim [etc.]: Bibliographisches Institut \& F.A. Brockhaus AG.

Duden-GW = Duden. 1976-1981. Das große Wörterbuch der deutschen Sprache in sechs Bänden. Hrsg. und bearb. vom Wissenschaftlichen Rat und den Mitarbeitern der Dudenredaktion unter der Leitung von Günther Drosdowski. Mannheim [etc.]: Bibliographisches Institut.

Duden-4${ }^{4}$ GFWB $=$ Wiss. Rat der Dudenredaktion (Hrsg.). 2007. Duden. Das große Fremdwörterbuch . Herkunft und Bedeutung der Fremdwörter. 4., aktual. Aufl. Mannheim [etc.]: Bibliographisches Institut \& F.A. Brockhaus A.G.

${ }^{2}$ Herders Sprachbuch $=1971$. Herders Sprachbuch. Ein neuer Weg zu gutem Deutsch. Freiburg [etc.]: Verlag Herder KG.

Schierholz, Stefan und Herbert Ernst Wiegand. 2005. Die Wörterbücher zur Sprach- und Kommunikationswissenschaft. Eine neue Konzeption der linguistischen Fachlexikographie und ihre computergestützte Praxis. Lexicographica 20: 164-264.

Steinhauer, Anja. 2005. Duden. Das Wörterbuch der Abkürzungen. Rund 50000 nationale und internationale Abkürzungen und Kurzwörter mit ihren Bedeutungen. 5., vollst. überarb. und erw. Aufl. Mannheim [etc.]: Bibliographisches Institut \& F.A. Brockhaus AG.

Wiegand, Herbert Ernst. 1998. Wörterbuchforschung. Untersuchungen zur Wörterbuchbenutzung, zur Theorie, Geschichte, Kritik und Automatisierung der Lexikographie. 1. Teilband. Mit 159 Illustrationen im Text. Berlin/New York: Walter de Gruyter. 
Wiegand, Herbert Ernst. 2002. Altes und Neues zur Mediostruktur in Printwörterbüchern. Lexicographica 18: 168-252.

Wiegand, Herbert Ernst. 2003. Überlegungen zur Typologie von Wörterbuchartikeln in Printwörterbüchern. Ein Beitrag zur Theorie der Wörterbuchform. Lexicographica 19: 169-313.

Wiegand, Herbert Ernst. 2005. Über die Datenakzessivität in Printwörterbüchern. Einblicke in neuere Entwicklungen einer Theorie der Wörterbuchform. Lexikos 15: 196-230.

Wiegand, Herbert Ernst. 2006. Das Lern- und Konsultationswörterbuch. Ein neuer Fachwörterbuchtyp am Beispiel der Wörterbücher zur Sprach- und Kommunikationswissenschaft (WSK). Lexikos 16: 205-221.

Wiegand, Herbert Ernst. 2006a. Die „Wörterbücher zur Sprach- und Kommunikationswissenschaft" (WSK) und ihre Benutzungsmöglichkeiten im Fach Deutsch als Fremdsprache. Dimova, Ana, Vida Jesenšek und Pavel Petkov (Hrsg.). 2006. Zweisprachige Lexikographie und Deutsch als Fremdsprache. Drittes Internationales Kolloquium zur Lexikographie und Wörterbuchforschung. Konstantin Preslavski-Universität Schumen, 23.-24. Oktober 2004: 1-35. Germanistische Linguistik 184-185. Hildesheim [etc.]: Georg Olms.

Wiegand, Herbert Ernst. 2007. Mittel zur Darstellung textueller Wörterbuchstrukturen von Printwörterbüchern. Ein Beitrag zur Methodologie der systematischen Wörterbuchforschung. Lexicographica 23. [Erscheint.]

WNED $=1993$. Webster's New Encyclopedic Dictionary. New York: Black Dog \& Leventhal Publishers Inc. 